EUROPEAN ORGANIZATION FOR NUCLEAR RESEARCH

CERN-PPE/94-127

3 August 1994

\title{
Performance of a Liquid Argon Accordion Hadronic Calorimeter Prototype
}

The RD3 collaboration

D.M.Gingrich, G.Greeniaus, P.Kitching, B.Olsen, J.L.Pinfold and N.L.Rodning Alberta University, Edmonton, Alberta, Canada

E.Boos and B.O.Schaoutnikov

Alma-Ata, Kazakhstan

B.Aubert, A.Bazan, B.Beaugiraud, J.Boniface, J.Colas, S.Jezequel, T.Leflour, M.Maire, F.Rival, M.Stipčević ${ }^{1}$, J.Thion, D.VanDenPlas,

I.Wingerter-Seez and Y.P.Zolnierowski

LAPP, Annecy, France

M.Chmeissani, E.Fernandez, L1.Garrido, M.Martinez and C.Padilla

Univ. Autònoma Barcelona, Bellaterra, Spain

H.A.Gordon, V.Radeka, D.Rahm, and D.Stephani

Brookhaven National Laboratory, Upton, USA

J.L.Chevalley, F.Gianotti ${ }^{2}$, M.Nessi, L.Poggioli and V.Vuillemin

CERN, Geneva, Switzerland

\footnotetext{
${ }^{1}$ On leave from Rudjer Bošković Institute, Zagreb, Croatia

${ }^{2}$ Also University of Milano, Italy
} 
J.M.Baze, L.Gosset, P.Lavocat, J.P.Lottin, B.Mansoulié, J.P.Meyer, J.F.Renardy, J.Schwindling and J.Teiger CEA, DSM/DAPNIA/SPP, CE Saclay, Gif-sur-Yvette, France

J.Collot, P.de Saintignon, D.Dzahini, J.Y.Hostachy, G.Laborie, G.Mahout, E.Merchez and J.Pouxe ISN, Grenoble, France

L.Hervas

Univ. Autònoma Madrid, Spain

A.Chekhtman, M.C.Cousinou, P.Dargent, B.Dinkespiler,

F.Etienne, P.Fassnacht, D.Fouchez, L.Martin, A.Miotto, E.Monnier, E.Nagy, C.Olivetto and S.Tisserant CPP Marseille, France

G.Battistoni, D.V.Camin, D.Cavalli, G.Costa, L.Cozzi, A.Cravero, N. Fedyakin, A.Ferrari, L.Mandelli, M.Mazzanti, L.Perini and P.Sala Dipartimento di Fisica dell'Università e Sezione INFN, Milano, Italy G.Azuelos, G.Beaudoin, P.Depommier, E.I.Florian, C.Leroy and J.Roy University of Montreal, Canada

E.Augé, R.Chase, J.C.Chollet, C.de La Taille, L.Fayard, D.Fournier A.Hirosoho, B.Merkel, J.M.Noppe, G.Parrour, P.Pétroff, A.Schaffer, N.Seguin, L.Serin and I.Vichou LAL, Orsay, France

O.Le Dortz, A.Savoy-Navarro, and P.Schwemling LPNHE, Universités de Paris VI et VII, France

L.O.Eek, B.Lund-Jensen and J.Soderqvist Royal Institute of Technology, Stockholm, Sweden

M.Lefebvre, S.Robertson and J.White University of Victoria, Canada 


\begin{abstract}
A liquid argon hadronic calorimeter using the "accordion" geometry and the electrostatic transformer readout scheme has been tested at CERN, together with a liquid argon accordion electromagnetic prototype. The results obtained for pions on the linearity, the energy resolution and the uniformity of the calorimeter response are well within the requirements for operation at the LHC.
\end{abstract}

(Submitted to Nuclear Instruments and Methods) 


\section{Introduction}

Calorimetry using liquid argon ( LAr) as an active medium is a well-proven technique at present colliders $[1,2]$. The intrinsic radiation resistance of this technique makes a LAr calorimeter a good candidate for a detector at the high luminosity Large Hadron Collider (LHC). However, operation at LHC requires the development of fast readout schemes.

The ionization electrons drift time through the liquid gap is rather long $(\simeq 450$ $\mathrm{ns}$ for a $2 \mathrm{~mm}$ argon gap) compared to the bunch crossing interval at LHC (25 ns), but a careful design of the signal collection path [3] should make it possible to detect the fast rise of the ionization current which contains all the information about the energy deposited into the liquid.

To this end, a new absorber and electrode geometry (the "accordion " geometry) has been designed and successfully tested with an electromagnetic (EM) calorimeter prototype [4]. This new geometry allows to reduce to a minimum the stray capacitances and inductances of electrical connections between sensitive gaps and readout electronics. It also allows an easy grouping, without cracks, of the accordion gaps into highly granular towers. In addition, this design is completely $\phi$ symmetric.

Typical cell capacitances of highly granular EM calorimeters $(\simeq 400 \mathrm{pF})$ are relatively easily matched to the input impedance of preamplifiers. The charge transfer time is fast enough $(\leq 10 \mathrm{~ns})$. This is no more true for the much larger cells of hadronic (HAD) calorimeters where large capacitances (several nanofarads) slow down the readout and cause large electronics noise. In absence of magnetic field, transformers can match the cell to the preamplifier input impedance. Increasing the number of preamplifiers reading a single calorimeter cell might also solve the difficulty. Some time ago, a technique known as the ElectroStatic Transformer (EST) readout scheme [5] has been developed. The gaps of a calorimetric cell are connected in series rather than in parallel as usual. To first order, the cell capacitance is the gap capacitance divided by the number of gaps in series instead of being multiplied by it. Thus the cell capacitance is much lower and the charge transfer time much shorter.

This paper describes the construction of a prototype of an hadronic LAr calorimeter which uses both the accordion and EST concepts. Simulation results and performances obtained in test beams at CERN are reported. 


\section{The Calorimeter Prototype}

The calorimeter prototype has been designed as a sector of a large cylindrical barrel calorimeter for an LHC experiment. Calorimeter cells are projective and the geometry is easily described by the radius $\mathrm{R}$, the rapidity $\eta$ and the azimuth angle $\phi$. In depth, a particle from the "interaction vertex" first hits at a radius of about $1.3 \mathrm{~m}$ an EM calorimeter, then at larger radii $(\simeq 2 \mathrm{~m}$ and $2.8 \mathrm{~m})$ two concentric hadronic modules.

The EM prototype is described in a separate paper [7]. It is built stacking 1.8 $\mathrm{mm}$ lead absorbers folded to an accordion shape interleaved by a kapton electrode and two $1.9 \mathrm{~mm}$ LAr gaps. It covers $27^{\circ}$ of azimuth and a rapidity interval from 0 to 1.1. It is divided into three readout samplings in depth. Each sampling is composed of fully pointing cells, with a granularity of $\Delta \eta \times \Delta \phi=0.018 \times 0.020$ in the two first samplings and $0.036 \times 0.020$ in the third one. The total thickness of the electromagnetic module is about 1.2 interaction lengths, and the total amount of material in front of the hadronic prototype, including the cryostat and the inactive liquid argon, is about 1.5 interaction lengths.

The hadronic part of the calorimeter is built in two separate modules. Each module has two samplings in depth, 1.2 and 1.6 absorption lengths deep respectively. Each hadronic module is eight towers wide in $\phi$ and nine (back sampling) or ten (front sampling) towers long in $\eta$. The total weight of the hadronic part is about 12 tons. The sampling fraction for muons is about $4.8 \%$.

\subsection{Mechanics of the Hadronic Prototype}

The hadronic prototype (figure 1) is made of $9.8 \mathrm{~mm}$ thick ground and readout stainless steel plates interleaved with $3 \mathrm{~mm}$ liquid argon gaps. Using a cold profiling technique, the converter plates are given an accordion shape with the folds parallel to the (fictitious) LHC beam axis. The amplitude of the waves has been chosen to correspond to the azimuthal size of one hadronic cell. The barrel cylindrical $\phi$ symmetry is obtained rotating subsequent plates with respect to the beam axis. In order to keep the liquid argon gap constant, the folding angle is changing along the radial (depth) dimension and each fold is given a curved shape (figure 2 ).

To get a constant amount of absorber (or liquid argon) seen by an incoming particle, independently of its azimuth, the overlap between accordion plates in the

prototype had to be optimized with a Monte Carlo simulation. For a simulation of $10 \mathrm{GeV}$ electrons and the geometry shown in figure 2, the energy deposited in the liquid argon is uniform to about $\pm 1 \%$ when changing the azimuth of the incident 
particle.

In order to get a total thickness of the hadronic part of about 5.6 interaction lengths while keeping the angles of the folds with respect to the incident particle direction of the order of $45^{\circ}$, the calorimeter is made of two modules in depth. To avoid doubling the cost of the profiling equipment for the prototype, the two modules were made of the same plates. Thus, only the first module was exactly projective in $\phi$.

After profiling, the readout plates were cut along projective $\eta$ lines and into two samplings in depth (figure 3) by using a water jet technique, while the ground plates were kept in one piece. Readout plates were then reassembled, like a jigsaw puzzle. The $5 \mathrm{~mm}$ thick space between two pieces of the puzzle, which was optimized for a low crosstalk of the electrostatic transformer readout, is filled with glass fibre wicks and epoxy resin.

The readout plates are then covered with a $140 \mu \mathrm{m}$ thick "prepreg" layer, a 75 $\mu \mathrm{m}$ thick insulating Kapton layer and a $150 \mu \mathrm{m}$ carbon loaded prepreg resistive layer. This resistive coating, cut into cells, is connected in the front (first sampling) or in the back (second sampling) to two high voltages busses (one for each side of the plate) running along the edges of the plates by resistive Kapton strips. The cladding (including the high voltage connection) is glued by polymerization to the plates by heating at $120^{\circ} \mathrm{C}$ under a 1 atm relative pressure during two hours.

A surface resistivity of $\simeq 300 \mathrm{k} \Omega / \square$ was chosen as a reasonable compromise for operation at the high luminosity of LHC. It is high enough to protect preamplifiers in case of accidental sparking and the voltage drop induced by the ionization current is low enough to not influence the charge collection in the gaps.

Before assembly, each plate was tested under a $4 \mathrm{kV}$ high voltage. No problems were found.

Plates are then assembled by grouping $5(7)$ readout plates and 2 ground plates in the first (second) module. The gaps are obtained by $3 \mathrm{~mm}$ thick insulating spacers. Six (resp. eight) liquid argon gaps are grouped to form projective towers in $\phi$, the $\eta$ granularity being obtained by the cutting of the readout plates. The tower size is $\Delta \eta \times \Delta \phi=0.045 \times 0.05$.

In the front and in the back of each module, a $1 \mathrm{~mm}$ thin stainless steel foil ("bubble shield") connects all ground plates together and closes the sensitive volume (fig. 2). This foil supports all preamplifiers and calibration circuits. 


\subsection{Implementation of the EST Readout Scheme}

As shown in figure 2, a calorimeter cell is enclosed between two ground plates and contains an odd number of readout plates. The structure is symmetric respective to the central plate which sums the signal coming from both sides. A $4 \mathrm{~cm}$ long, 1 $\mathrm{cm}$ wide copper clad Kapton strip connects the stainless steel of the central readout plate to the preamplifier input. The other readout plates only capacitively transfer the signal to the central one without any special connection. The signal return path, through the "bubble shield" and the ground plates, is very short and presents a negligible inductance.

The high voltage distribution (figure 4) ensures that the electric field points in the same direction for all gaps on one side of the central electrode and in the reverse direction on the other side. On each side of the central electrode, gaps are connected in series; the two sides are in parallel. The number of gaps in series (EST ratio) is 3 in the first two samplings and 4 in the last two.

A crosstalk capacitance exists between adjacent tiles (in the eta direction) of a same absorber plate and limits the performance of the EST structure. The crosstalk between two tiles of a same module adjacent in depth is much smaller because of the smaller common area and the slightly bigger gaps between the tiles. The crosstalk capacitances increase the cell capacitance, which is no longer the gap capacitance divided by the EST ratio and multiplied by the number of gaps in parallel. Typical values for the prototype, as obtained from the simulation described below, are given in table 1 . The effect of these crosstalk capacitances are discussed in section 3 below.

\section{$2.3 \quad$ Readout Electronics}

The 304 calorimeter cells are read out by GaAs MESFETs hybrid charge preamplifiers located in the cold very close to the cells. The feedback network is made of an $8.2 \mathrm{pF}$ capacitance in parallel with a $330 \mathrm{k} \Omega$ resistor. With such a feedback capacitance value, the input resistance of the preamplifier is $30 \Omega$ and its input capacitance is $60 \mathrm{pF}$. The equivalent noise charge (ENC) at a $100 \mathrm{~ns}$ peaking time response to a delta pulse is $\mathrm{ENC}=600 \mathrm{e}+10 \mathrm{e} / \mathrm{pF}$. The power dissipation is 54 $\mathrm{mW}$ per channel [6].

Cables $(50 \Omega)$ bring the signals outside of the cryostat to CR2RC3 shapers. Simulation of the pile-up noise expected at the LHC luminosity in the hadronic modules led to choose the shaper time constants such that a current impulse produces a signal with a maximum after $90 \mathrm{~ns}$. For this value, the quadratic sum of the pileup and electronics noises is minimum and the maximum of the triangle signal from the 
calorimeter is at about $180 \mathrm{~ns}$. This peak value is digitized with a 12 bit ADC and gives the energy measurement.

Precision resistors $(50 \mathrm{k} \Omega$ ) located close to the cells are used to inject exponential calibration current pulses [7].

\section{Simulation}

A Monte Carlo simulation of the calorimeter reproducing the experimental setup has been performed with GEANT 3.159 [11]. In the simulation, the EM part of the calorimeter was described by parallel plates with thicknesses multiplied by a factor $\sqrt{2}$ whereas the hadronic calorimeter is fully described with accordion-shaped plates. The showering process was followed down to a cut-off energy of $500 \mathrm{KeV}$ since no improvement on the energy resolution has been observed by going to $100 \mathrm{KeV}$. Both GHEISHA and FLUKA hadronic shower packages were used. When comparing simulation results with test beam data, incoherent and coherent noises, as measured on random trigger events in the data, have been added. The beam spread is also simulated.

In order to evaluate the EST crosstalk effect on the energy measurement, a simulation of the EST readout has been performed and implemented in some of the GEANT simulations and is described below. The effects of the non uniform electric field and larger drift time in the corners of the accordion folds were not simulated.

\subsection{Charge Delocalization due to EST Crosstalk Effect}

The mechanical design, with ground plates in between cells of different azimuth, guarantees that there is no crosstalk in the $\phi$ direction. However, in $\eta$, two tiles of the same plate are capacitively coupled as discussed in 2.2 above. Thus, the electric circuit in figure 5 is used to represent half a calorimeter cell with $N$ gaps of capacitance $C$. The $N$ gaps are connected in series (as required by the EST readout scheme) and the current of each gap is summed at the input port of a common charge amplifier. To the same circuit, an additional capacitive coupling $c$ was introduced feeding signal to adjacent gaps in order to simulate the crosstalk induced by the EST readout scheme. Since the EST crosstalk is expected to have negligible effects on cells which are not directly adjacent to the central one, only three cells have been considered : the central one and the two adjacent cells in $\eta$, lumped into one larger capacitance tower. 
The analysis of this model when current impulses are applied to a cell gap reveals the main features of the EST readout [8] :

- The time constant describing signal collection is $R C / N$, much faster than with $N$ gaps connected in parallel $(R C \times N)$.

- When the same current is applied to all gaps, the EST structure introduces no additional crosstalk as no net charge accumulates on the transfer electrodes.

- A crosstalk signal appears when gap currents are unbalanced. This signal can be positive or negative. However summing over the main cell and its neighbours, crosstalk signals cancel out and the amplitude of the signal without crosstalk is recovered. Energy is not lost but only "delocalized". Normalized weights can thus be defined to describe signal sharing between adjacent cells.

Then, to take into account the triangle shape of the input currents (the drift time was taken as $700 \mathrm{~ns}$ corresponding to the $3 \mathrm{~mm}$ argon gap) and the subsequent stages of pre amplification and shaping, a full SPICE simulation of the signal collection was

performed [9]. Figure 6 shows simulated signals when all gaps are pulsed uniformly. At measurement time, the crosstalk is small $(\simeq 1 \%)$. It can be much larger $(\simeq 40 \%)$ when only one gap is pulsed (figure 7).

Table 2 gives the fraction of signal seen by the central cell $\left(P_{C}\right)$ and its two neighbours in $\eta\left(P_{A}\right)$ when a current is injected in one of the gaps of the central cell.

\subsection{EST Influence on Signals from Hadronic Showers}

The GHEISHA and FLUKA hadronic shower packages were used together with GEANT to generate two samples of 500 events corresponding to $100 \mathrm{GeV}$ charged pions hitting the calorimeter prototype at different places in its central region. The beam spot was about one EM cell wide.

For each sample, the measurement of the energy deposited in the hadronic calorimeter cells by pions was evaluated with and without the EST crosstalk effect. Without the EST effect, the energy deposited by a pion in a hadronic cell is just the sum of the energies measured in each gap of this cell.

When the EST crosstalk effect is taken into account, the energy measured depends not only on the energies collected in each gap of the cell, but also on the energies collected by the gaps of neighbouring cells in $\eta$, according to the weights given in table 2 . 
Since the EST crosstalk is expected to have no effect in $\phi$, one of the most sensitive quantities is the transverse profile in the $\eta$ direction. Figure 8 shows this profile in the first hadronic sampling with and without EST for the GEANT-GHEISHA simulation. The EST effect is negligible. The same conclusion holds for the GEANTFLUKA simulation. This indicates that, with the cell size of the prototype and the accordion geometry, the energy deposition in an hadronic shower is uniform over one cell.

The effect of the EST readout on the energy resolution has also been measured and will be described below.

\section{Test Beam Results}

\subsection{Test Beam Layout}

The test calorimeter has been exposed to beams of muons, electrons and charged pions of energies ranging from 20 to $400 \mathrm{GeV}$ at the CERN H8 beam facility. The beam line was instrumented with :

- a converter made of $3 \mathrm{X}_{0}$ of lead located far upstream, in front of a bending magnet. This converter, used during pion exposure, removes most of the electrons.

- two trigger counters $\left(2.5 \times 2.5 \mathrm{~cm}^{2}\right.$.

- three delay line wire chambers to measure the incoming particle position and direction.

- a "preshower" made of a $1 \mathrm{X}_{0}$ lead sheet in front of a scintillator counter to reject the remaining electron contamination.

The hadronic prototype was placed behind the EM accordion prototype, in the same cryostat. The cryostat was mounted on a support allowing rotations in $\phi$ and $\eta$ with respect to the fictitious LHC interaction point. This was used to scan the front surface of the calorimeter.

For the pion and the muon data analyses, cuts on the time information from the wire chambers were performed to remove events with two particles or events with an early shower. Particles far from the nominal beam position were also removed. 


\subsection{Noise Measurement and Muon Data Analysis}

The noise has been measured by looking at the energy seen in regions of various sizes in random trigger events. The noise is the quadratic sum of random and coherent noises. Typical random noise values are between 50 and $90 \mathrm{MeV}$ per cell. Most of the coherent noise was traced to a faulty high voltage supply. Its value ranges from 4 to $13 \mathrm{MeV}$ per cell.

In order to study the response of the calorimeter to muons, a precise alignment of the beam chambers and the calorimeters was performed by using electron and pion data. Muon events were selected from a $180 \mathrm{GeV}$ tertiary beam by requiring no more than $10 \mathrm{GeV}$ energy deposited in the calorimeters.

Because of the accordion shape of the calorimeter, two towers in $\phi$ were considered to measure the muon energy deposition. Using the track reconstructed from the beam chambers, a fiducial volume within these two towers was defined and muons not in this volume were rejected. The electronic noise was measured for the same group of cells using random trigger events.

Figure 9 shows the energy deposited in the first sampling of the hadronic calorimeter for muons and the energy computed for random trigger events. To define the energy scale, the calibration factors for high energy densities, obtained by the weighting method described in the next section, were used. Figure 10 shows the muon signal for the full hadronic calorimeter. The signal over noise ratio is 6.3 for the first sampling of the hadronic calorimeter and 7.9 when the full calorimeter is used.

The energy scale for the Monte Carlo simulation has been adjusted so that the peak position in the full hadronic calorimeter is the same as in the data. Figures 9 and 10 show that the shape and the tail of the energy distributions are well reproduced by the simulation.

\subsection{Pion Data Analysis}

\subsubsection{Hadronic Shower Profiles and Energy Leakage}

The study of the shower sizes is interesting to get an idea of lateral and longitudinal leakages in the size-limited hadronic prototype and to check whether the EST readout induces any widening of the apparent transverse size in the data.

Figure 11 shows the $\eta$ transverse profile, in the first hadronic sampling, for 100 $\mathrm{GeV}$ pions in the data and for the GEANT-GHEISHA simulation. The profile from 
GEANT-GHEISHA is slightly narrower than the data whereas the profile from the GEANT-FLUKA simulation is slightly wider. There is no evidence of any large widening of the shower size in the data due to the EST. Because of the accordion shape of the absorbers, the energy profile in the $\phi$ direction is less sensitive to small differences between the simulations or between the simulations and the data. Figure 12 show that GEANT-GHEISHA is in correct agreement with the data in the $\phi$ direction, and so is GEANT-FLUKA. The simulation predicts a lateral leakage of about $1 \%$ in the $\phi$ direction.

Figure 13 shows the fraction of the measured energy in each of the four hadronic samplings for $400 \mathrm{GeV}$ pions interacting in the first hadronic sampling. These events have been selected by cuts on the energy measured in the EM calorimeter and in the first hadronic module. The agreement between the data and the Monte Carlo simulations gives reasonable confidence in the measurement of longitudinal leakage behind the calorimeter with the simulation. Table 3 summarizes the lateral and longitudinal leakages in the Monte Carlo simulation. At $400 \mathrm{GeV}$, more than $4 \%$ of the energy is lost behind the 7 interaction lengths thick calorimeter. The energy lost by leakage can be recovered, on average, by the energy reconstruction described below, but fluctuations in the leakage will contribute to the constant term in the energy resolution.

\subsubsection{Energy Reconstruction}

It is now well established that a lead-iron-LAr calorimeter is non compensating, i.e. the signals from electrons are on average higher than those from pions of the same energy. In RD3, the ratio $c_{\mathrm{EM}}^{\pi} / c_{\mathrm{EM}}^{e}$ of the calibration constants of the EM part of the calorimeter for pions and electrons when the total energy is defined as $\tilde{E}=c_{\mathrm{EM}} E_{\mathrm{EM}}+$ $c_{\mathrm{HAD}} E_{\mathrm{HAD}}$ was found to vary from 1.4 at $20 \mathrm{GeV}$ to 1.1 at $400 \mathrm{GeV}$. However, as successfully shown by the H1 collaboration [2], the high granularity of the calorimeter helps to distinguish electromagnetic and purely hadronic shower components and to reconstruct the hadronic energies by applying weighting functions.

The study of energy reconstruction is done using data samples of several thousand events each, on eight beam energies $(20,30,50,100,150,200,300,400 \mathrm{GeV})$ and several Monte Carlo data samples using both GHEISHA and FLUKA packages. The data were taken in the centre of the calorimeter and the simulation was performed at the same point.

The measurement of a shower energy is performed in an area of $11 \times 11$ cells in the EM module and $7 \times 7$ cells in the hadronic modules, around the beam direction. In order to improve the signal-to-noise ratio, only cells with absolute signals greater than 1.3 times their measured electronics noise (RMS) value are used. This cut 
removes $80 \%$ of empty cells and keeps in average, according to the simulation, $96 \%$ of the signal energy for $100 \mathrm{GeV}$ pions. The cut is done on the absolute value of the signal to ensure that the average energy of random trigger events and the average energy of empty cells in pion events are kept at zero.

The cell energy density $\rho=$ (cell energy) / (cell volume), where "cell volume" is the volume divided by the volume of the smallest cell, is used to distinguish between mainly EM energy deposition and mainly hadronic ones. Figure 14 shows the distribution of this variable in the hadronic part of the calorimeter. Negative values are due to electronics noise. Simulations have shown that the EST contribution to those negative values is small. High energy density values are due to $\pi^{0}$ 's produced during hadronic interactions. Indeed, the effective radiation length of the calorimeter $(\simeq$ $24 \mathrm{~mm})$ is much shorter than the effective interaction length $(\simeq 220 \mathrm{~mm})$, and so $\pi^{0}$ 's deposit most of their energy in a single hadronic cell.

As the intrinsic $e / h$ ratio is greater than one, fluctuations in the electromagnetic share in the total shower energy worsen the energy resolution and cause a nonlinear calorimeter response. These effects are partly corrected for by using weights which depend on the cell energy densities. We use two weighting functions, one for the EM part and one for the HAD part of the calorimeter, as the structure of these two parts is very different. They are :

$$
\begin{array}{r}
w_{\mathrm{EM}}(\boldsymbol{\theta}, \rho)=\left(1-\mathrm{e}^{-|\rho| / e_{1}}\right) \cdot\left(e_{2} \cdot \mathrm{e}^{-|\rho| / e_{3}}+\epsilon_{4}\right) \\
w_{\mathrm{HAD}}(\boldsymbol{\theta}, \rho)=\left(1-\mathrm{e}^{-|\rho| / h_{1}}\right) \cdot\left(h_{2} \cdot \mathrm{e}^{-|\rho| / h_{3}}+h_{4}\right)
\end{array}
$$

where $\boldsymbol{\theta}=\left(e_{1}, e_{2}, e_{3}, e_{4}, h_{1}, h_{2}, h_{3}, h_{4}\right)$ is the vector of function parameters. In reference [10] a study with discrete weights was also done and results obtained justify the shape of the weighting functions used in this analysis. At high energy density, weights approach the calibration factor for electromagnetic showers $\left(e_{4}, h_{4}\right)$. They rise for lower $\rho$ values to compensate for the invisible energy in the purely hadronic component of the shower, which dominates this region of low energy density. The contribution of cells with very small signals is suppressed by the weighting functions which go to zero. Indeed, these cells mainly add noise.

The particle energy is estimated as :

$$
\tilde{E}=\sum_{k=1}^{3} c_{k} \sum_{j \in(\mathrm{EM})_{k}} w_{\mathrm{EM}}\left(\boldsymbol{\theta},\left|\rho_{j}\right|\right) \cdot E_{j}+\sum_{k=4}^{7} c_{k} \sum_{j \epsilon(\mathrm{HAD})_{k}} w_{\mathrm{HAD}}\left(\boldsymbol{\theta},\left|\rho_{j}\right|\right) \cdot E_{j}
$$

The parameters $\boldsymbol{\theta}$ and calibration factors $c_{k}$ are determined by minimization of the following functional:

$$
\frac{1}{N_{\mathrm{EVT}}} \sum_{i=1}^{N_{\mathrm{EVT}}}\left(\tilde{E}_{i}-E_{\mathrm{BEAM}}\right)^{2}=\min .
$$


where $E_{\mathrm{BEAM}}$ is the known beam energy, $N_{\mathrm{EVT}}$ is the number of events. This minimization method introduces a small negative bias [12] which is corrected for, at each beam energy, by multiplying the factors $e_{2}, e_{4}, h_{2}$ and $h_{4}$ by $E_{B E A M} /<\tilde{E}>$, where $\langle\tilde{E}\rangle$ is the gaussian fitted average energy.

After this rescaling, the calibration factors $c_{1}, \ldots, c_{6}$ are stable with beam energy and therefore they have been fixed. Factor $c_{7}$ rises by about $30 \%$ from $20 \mathrm{GeV}$ to $400 \mathrm{GeV}$. This is understood as a way to compensate for leakage at the back of the calorimeter.

Parameters of the weighting functions $e_{1}, \ldots, e_{4}, h_{1}, \ldots, h_{4}$ also depend on beam energy (Fig. 15). However, this dependence is rather smooth and easily parameterized by linear or exponential functions. In accordance to its interpretation as calibration factor for electromagnetic showers, the parameter $h_{4}$ (as well as $e_{4}$ ) is almost energy independent. Figure 16 shows the evolution of the hadronic weighting function from 30 to $300 \mathrm{GeV}$. We see that the weighting function goes to the limiting value $\left(h_{4}\right)$ slower and slower as the beam energy rises, which means that the average electromagnetic energy density of a hadronic shower rises with energy. The noise-reducing parameters $e_{1}$ and $h_{1}$ are small (and positive) as most of the empty cells are already excluded by the noise cut mentioned before.

The energy reconstruction is done in the following manner. A first estimate of the energy is computed as the sum of signals over the calorimeter cells and is used to calculate the weighting parameters. A new weighted energy is then estimated and the procedure is iterated four times, no improvement being seen with more than four iterations.

The same minimization was performed on the Monte Carlo simulated events, and the weights obtained were found in agreement with the data.

\subsubsection{Energy Response and Resolution}

To estimate the calorimeter performance on energy measurement using the method above, another set of events, not used to measure the weighting functions, was processed with the algorithm just described.

Figure 17 shows the reconstructed energy distribution for $100 \mathrm{GeV}$ pions, in data and in the GHEISHA Monte Carlo simulation. The energy resolution is 6.5 $\mathrm{GeV}$. The small tail of low energy events is mainly due to non complete shower containment. Simulation and data are in good agreement.

Figure 18 shows the difference between the average reconstructed energy and the 
nominal beam energy as a function of beam energy. The calorimeter is calibrated to better than $1 \%$ RMS over the full energy range $20 \mathrm{GeV}-400 \mathrm{GeV}$.

As the calorimeter response is linear and nearly gaussian (after weighting), its resolution is defined as the standard deviation of a gaussian fitted to the reconstructed energy distribution divided by its mean value.

The energy resolution as a function of the beam energy is shown in figure 19 for the data and the GHEISHA and FLUKA simulations. The resolution of the data is well fitted by:

$$
\sigma / E=\frac{(51.6 \pm 2.6) \%}{\sqrt{E}} \oplus(3.3 \pm 0.1) \% \oplus \frac{2.5 \pm 0.1}{E}(E \text { in } \mathrm{GeV})
$$

The fitted "noise" term $(2.5 \mathrm{GeV})$ is in agreement with the quadratic sum of the electronic noise $(1.8 \mathrm{GeV})$ and the RMS energy loss $(1.5 \mathrm{GeV})$ due to the 1.3 sigma cut. As discussed above, part of the electronic noise was due to a faulty high voltage supply. With this problem cured, the electronic noise would be $1.3 \mathrm{GeV}$ and the 1/E term would become $2 \mathrm{GeV} / \mathrm{E}$. Simulation and further analysis of the data show that a significant fraction of the constant term comes from longitudinal leakage.

The effect of the EST on the resolution is small. In the GEANT-GHEISHA simulation, the resolution is $(7.0 \pm 0.2) \%$ at $100 \mathrm{GeV}$ if currents from all the gaps are just summed up, and $(7.4 \pm 0.2) \%$ if EST readout scheme and all mutual capacitances are invoked.

The weighting procedure is quite effective in improving the energy resolution: if, rather than the weighting procedure, only two calibration constants are used (one for the electromagnetic calorimeter and one for the hadronic calorimeter), the resolution is $12.2 \%$ instead of $6.5 \%$, at $100 \mathrm{GeV}$ in the data.

Figure 19 also shows the expected resolution from Monte Carlo simulations. Although both GHEISHA and FLUKA are in reasonable agreement with the data for the shower profiles, FLUKA predicts a resolution better, by 10 to $20 \%$, than the resolution obtained in the data, for energies below $200 \mathrm{GeV}$.

\subsubsection{Uniformity}

Since azimuthal and rapidity coverage of the test calorimeter are just enough for a good pion measurement when the beam is directed to the middle of the calorimeter, there is little room for a uniformity scan. Nevertheless, such a scan was made by 
moving the beam along the $\eta$ direction over 4 middle cells of the hadronic calorimeter in 5 fine steps. The scan line is one hadronic cell away in azimuth from the position of the energy scan discussed above. Figure 20 shows the result of the scan with 200 $\mathrm{GeV}$ pions. The abscissa of this graph is expressed in electromagnetic cell numbers. Despite the reconstruction is done with the weights previously determined during the energy scan at another position, the homogenity is very good $( \pm 1 \%)$.

\subsubsection{High Voltage Dependence}

The operating high voltage was $2 \mathrm{kV}$ for the e.m. module and $3 \mathrm{kV}$ for the two hadronic ones i.e. a drift field of $\simeq 10 \mathrm{kV} / \mathrm{cm}$. The calorimeter was also tested at lower high voltage. At $2000 \mathrm{~V}$ on all hadronic modules, the signal drops by $15 \%$, but, after calibration, the energy resolution is recovered.

\section{Conclusion}

A liquid argon hadronic calorimeter prototype using the accordion geometry for the converter plates and the electrostatic transformer readout scheme has been built and tested, together with a liquid argon accordion electromagnetic prototype.

The electrostatic transformer readout, which is used to match the calorimeter cell capacitance to the input impedance of the preamplifiers and allows a fast readout, is not expected to degrade the performance of the calorimeter. No effect is seen in the data.

As the $\mathrm{e} / \pi$ ratio is greater than one, a weighting procedure is used to measure the energy of pions. Weights are determined from measured quantities only. A significant improvement is obtained with the weighting procedure rather than a simple calibration. For beam energies between 20 and $400 \mathrm{GeV}$, the energy resolution for pions is:

$$
\sigma / E=\frac{(51.6 \pm 2.6) \%}{\sqrt{E}} \oplus(3.3 \pm 0.1) \% \oplus \frac{2.5 \pm 0.1}{E}(E \text { in } \mathrm{GeV})
$$

Performances on linearity and uniformity are also well within requirements for operation at a high luminosity high energy collider.

The resolution measured in the data is in agreement with a GEANT Monte Carlo 
simulation using the GHEISHA hadronic shower package, whereas the GEANTFLUKA simulation predicts a resolution 10 to $20 \%$ better.

\section{Acknowledgements}

We are indebted to the technical and support staffs of the participating laboratories. We would also like to thank the AQUARESE ${ }^{3}$, COTTON ${ }^{4}$ and PROFILAFROID ${ }^{5} \mathrm{com}-$ panies for their work on the prototype.

\footnotetext{
${ }^{3}$ AQUARESE, 1 Allee des Garays, 91120 Palaiseau, FRANCE

${ }^{4}$ COTTON, La Sône $38160 S^{t}$ Marcellin, FRANCE

${ }^{5}$ PROFILAFROID, 60390 Bailleul sur Therain, FRANCE
} 


\section{References}

[1] M. Abolins et al. (The D0 collaboration) Nucl. Instr. and Meth. A280 (1989) 36.

[2] W. Braunschweig et al. (H1 calorimeter group), Nucl. Instr. and Meth. A265 (1988) 419, A275 (1989) 246, Report DESY 89-022 (1989).

B. Andrieu et al. (H1 calorimeter group), report DESY 93-047.

[3] V. Radeka and S. Rescia Nucl. Instr. and Meth. A265 (1988) 228.

[4] B. Aubert et al. (The RD3 collaboration) Nucl. Instr. and Meth. A309 (1991) 438, A321 (1992) 467, A325 (1993) 116.

[5] J. Colas, M. Pripstein and W.A. Wenzel: The Electostatic Transformer, Nucl. Instr. and Meth. A294 (1990) 583.

[6] D.V. Camin, G. Pessina and E. Previtali: Front-end in GaAs, Nucl. Instr. and Meth. A315 (1992) 385.

[7] The RD3 collaboration, to be published in Nucl. Instr. and Meth.

[8] Analytical Description of an EST, J. Colas, W.A. Wenzel, WALIC note N-2789, LAPP-EXP-90.07

[9] F. Rival, Thèse, Université de Savoie, 1994

[10] M. Stipčević, Thèse, Université de Savoie, 1994

[11] GEANT User's Guide, CERN DD/78/2

[12] D. Lincoln, G. Morrow and P. Kasper: A hidden bias in a common calorimeter calibration scheme, Nucl. Instr. and Meth. A345 (1994) 449.

\section{List of Figures}

1 The hadronic calorimeter prototype is built from two independent modules. Each module has two samplings in depth with readout electronics located on the front and back faces. Clearly visible on this picture are the "accordion" shaped absorbers and the eight row of cells in the azimuth. . . . . . . . . . . . . 22

2 R- $\phi$ view of two calorimeter cells from module 1 showing the two ground plates at the outside, the transfer plates and the central readout plate connected to preamplifiers located on the "bubble shields". 
$3 \quad \mathrm{R}-\eta$ view of calorimeter cells showing the calorimeter depth segmentation and $\eta$ granularity. . . . . . . . . . . . . . . . 2

4 Schematic of an EST cell with a transformer ratio 3. The high voltage distribution ensures that the signal is collected on the central readout electrode. . . . . . . . . . . . . . . 25

$5 \quad$ Electrical circuit showing a half-cell with its gaps and its readout preamplifier. This central cell is connected through crosstalk capaci-

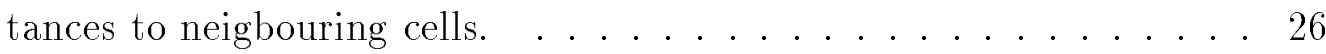

6 Simulated central tower signal and crosstalk signal in one of the adjacent cell when all gaps see the same energy deposition. Note the change of scale between the two pulses. . . . . . . . . . 27

7 Simulated central tower signal and crosstalk signal in one of the adjacent cell when energy is only deposited in the gap shown. Note the change of scale between the two pulses. . . . . . . . . . 28

8 Transverse energy distribution in the $\eta$ direction for $100 \mathrm{GeV}$ pions in the GEANT-GHEISHA simulation with and without EST crosstalk.

$9 \quad$ Energy distribution in the first sampling of the hadronic calorimeter for muons and for random trigger events. . . . . . . . . 30

10 Energy distribution in the hadronic calorimeter over the four samplings in depth for muons and for random trigger events. . . . . . . 31

11 Transverse energy distribution in the $\eta$ direction for $100 \mathrm{GeV}$ pions. 32

12 Transverse energy distribution in the $\phi$ direction for $100 \mathrm{GeV}$ pions. 33

13 Longitudinal profile for $400 \mathrm{GeV}$ pions interacting in the first hadronic sampling. . . . . . . . . . . . . . . 34

14 Distribution of the cell energy density $\rho$ for $100 \mathrm{GeV}$ pions. . . . . 35

15 Parameters of the weighting function $w_{h}$ as a function of beam energy. $\mathrm{h} 1$ and $\mathrm{h} 3$ are in GeV. ....................... 36

16 Weighting function $w_{H A D}(\boldsymbol{\theta}, \rho)$ in hadronic modules for several beam

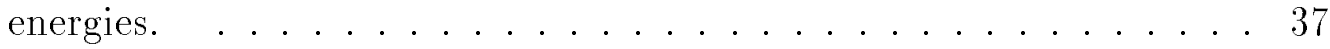


17 Distribution of the reconstructed energy for $100 \mathrm{GeV}$ pions. The histogram represents the data, dots are the GEANT-GHEISHA Monte Carlo simulation results. . . . . . . . . . . . . 38

18 Relative difference between reconstructed energy and beam energy (pion data). . . . . . . . . . . . . . 39

19 Energy resolution as a function of the beam energy (calibration + weighting $\ldots \ldots \ldots \ldots \ldots \ldots \ldots \ldots$

20 Uniformity of the calorimeter response as a function of impact point

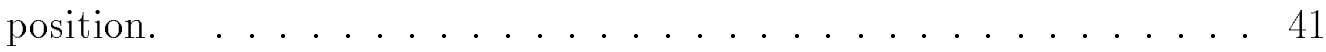




\begin{tabular}{|c|c|c|c|c|}
\hline Sampling \# & $\begin{array}{c}\text { EST } \\
\text { ratio }\end{array}$ & $\begin{array}{c}\text { cell } \\
\text { capacitance } \\
(\mathrm{pF})\end{array}$ & $\begin{array}{c}\text { gap } \\
\text { capacitance } \\
(\mathrm{pF})\end{array}$ & $\begin{array}{c}\text { crosstalk } \\
\text { capacitance } \\
(\mathrm{pF})\end{array}$ \\
\hline 1 & 3 & 148 & 131 & 40 \\
2 & 3 & 229 & 205 & 60 \\
3 & 4 & 157 & 182 & 40 \\
4 & 4 & 236 & 274 & 60 \\
\hline
\end{tabular}

Table 1: Typical cell, gap and crosstalk capacitances values (pF).

\begin{tabular}{|c|c|c|c|c|}
\cline { 2 - 5 } \multicolumn{1}{c|}{} & \multicolumn{2}{c|}{ first module } & \multicolumn{2}{c|}{ second module } \\
\hline $\begin{array}{c}\text { gap of } \\
\text { injection }\end{array}$ & central & left $/$ right & central & left/right \\
cell $\left.P_{C}\right)$ & cells $\left(P_{A}\right)$ & cell $\left(P_{C}\right)$ & cells $\left(P_{A}\right)$ \\
\hline 1 & 0.596 & 0.202 & 0.522 & 0.239 \\
2 & 0.832 & 0.084 & 0.648 & 0.176 \\
3 & 1.532 & -0.266 & 0.989 & 0.006 \\
4 & - & - & 1.782 & -0.391 \\
\hline
\end{tabular}

Table 2: Fraction of signal seen in each cell when energy is injected in one gap of the central cell. Gap 1 is near the ground plate and gap 3 (in module 1) or 4 (in module 2) is near the readout plate.

\begin{tabular}{|c|c|c|c|c|}
\cline { 2 - 5 } \multicolumn{1}{c|}{} & \multicolumn{3}{c|}{ leakage $(\mathrm{GeV})$} \\
\hline$E_{\text {beam }}(\mathrm{GeV})$ & \multicolumn{2}{|c|}{ longitudinal } & \multicolumn{2}{c|}{ lateral } \\
\hline & $<>$ & RMS & $<>$ & RMS \\
20 & 0.28 & 1.5 & 0.25 & 0.22 \\
100 & 2.2 & 5.3 & 1.1 & 0.62 \\
200 & 7.1 & 15.1 & 2.0 & 0.87 \\
400 & 17.1 & 34.5 & 4.0 & 1.58 \\
\hline
\end{tabular}

Table 3: Longitudinal and lateral leakages in the GEANT-GHEISHA Monte Carlo simulation. 


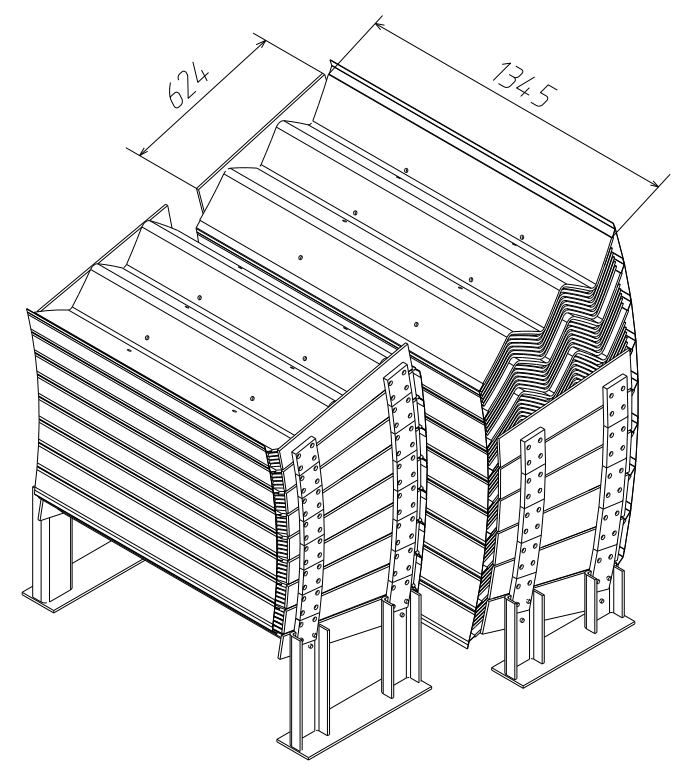

Figure 1: The hadronic calorimeter prototype is built from two independent modules. Each module has two samplings in depth with readout electronics located on the front and back faces. Clearly visible on this picture are the "accordion" shaped absorbers and the eight row of cells in the azimuth. 


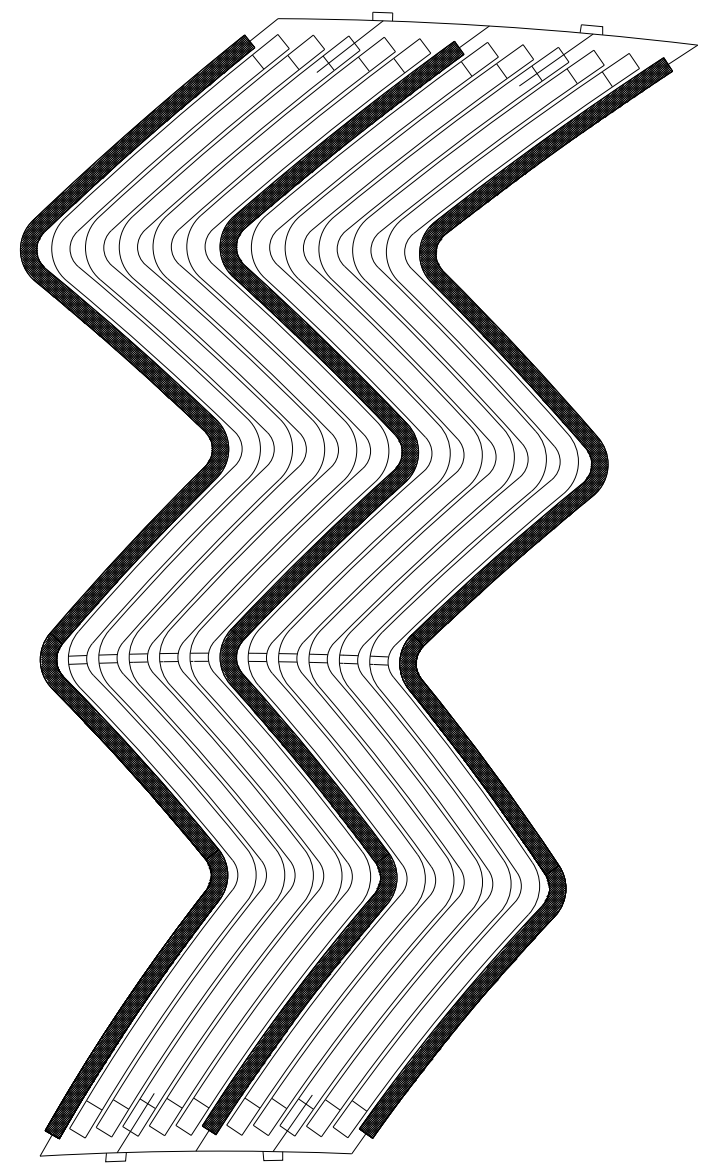

Figure 2: $\mathrm{R}-\phi$ view of two calorimeter cells from module 1 showing the two ground plates at the outside, the transfer plates and the central readout plate connected to preamplifiers located on the "bubble shields". 


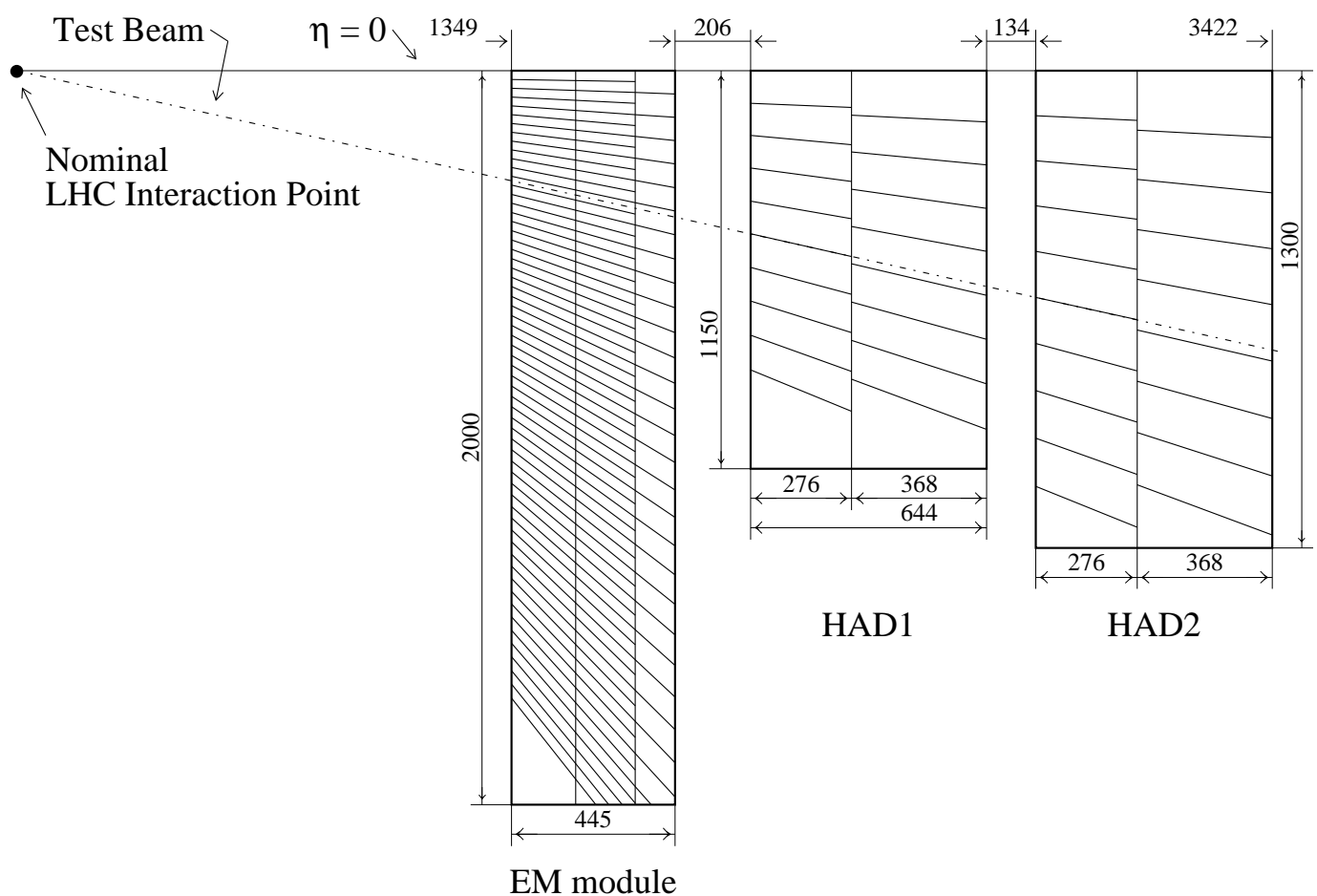

Figure 3: R- $\eta$ view of calorimeter cells showing the calorimeter depth segmentation and $\eta$ granularity. 


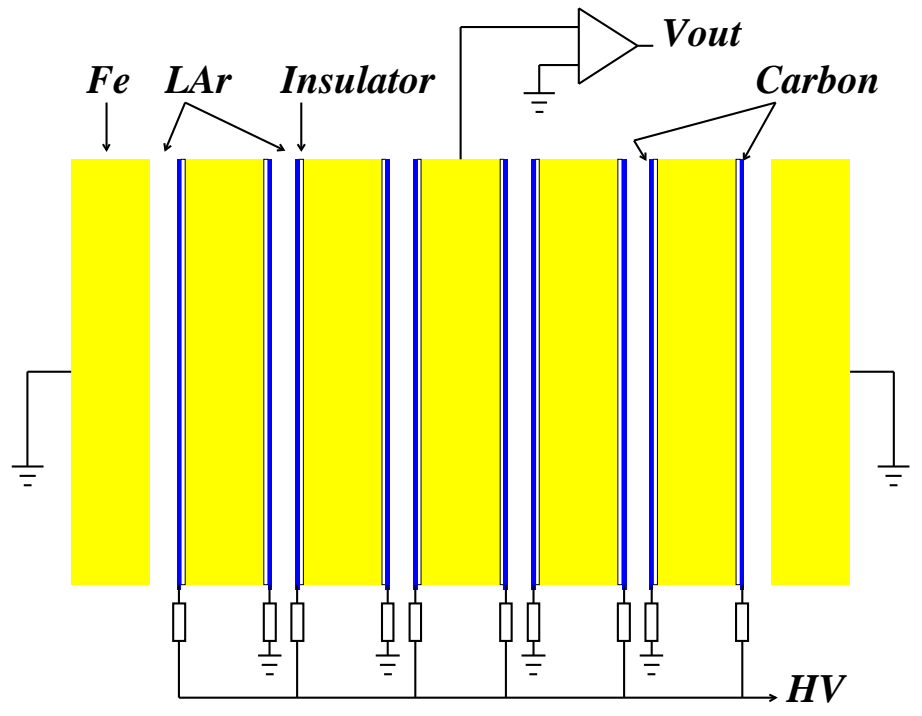

Figure 4: Schematic of an EST cell with a transformer ratio 3. The high voltage distribution ensures that the signal is collected on the central readout electrode. 


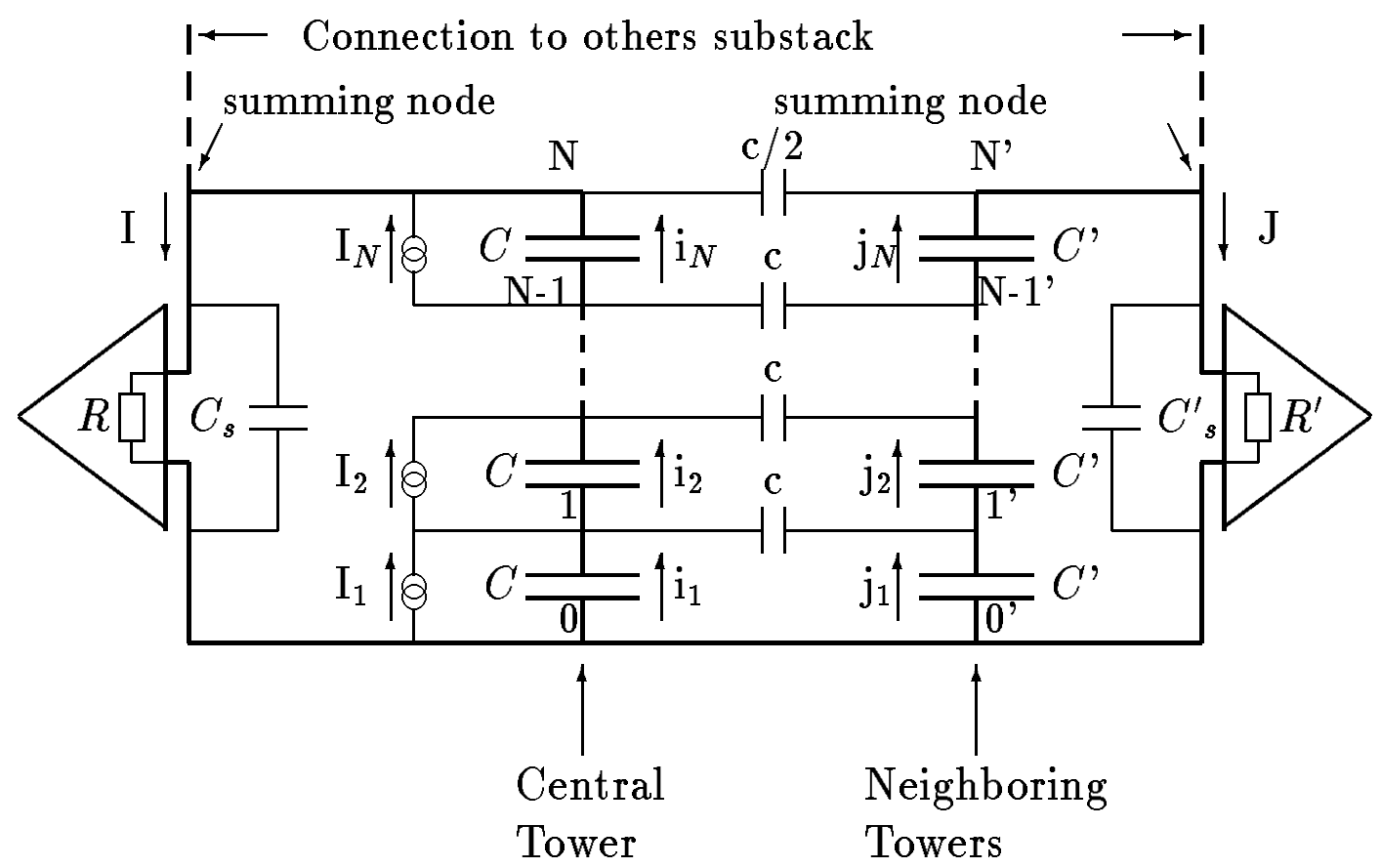

Figure 5: Electrical circuit showing a half-cell with its gaps and its readout preamplifier. This central cell is connected through crosstalk capacitances to neigbouring cells. 


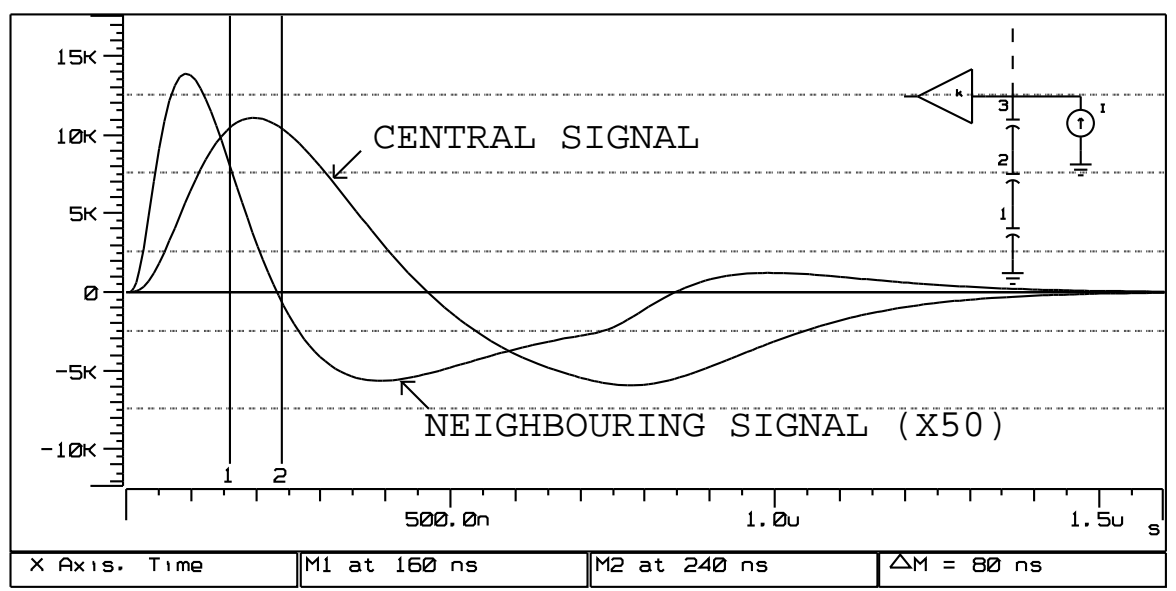

Figure 6: Simulated central tower signal and crosstalk signal in one of the adjacent cell when all gaps see the same energy deposition. Note the change of scale between the two pulses. 


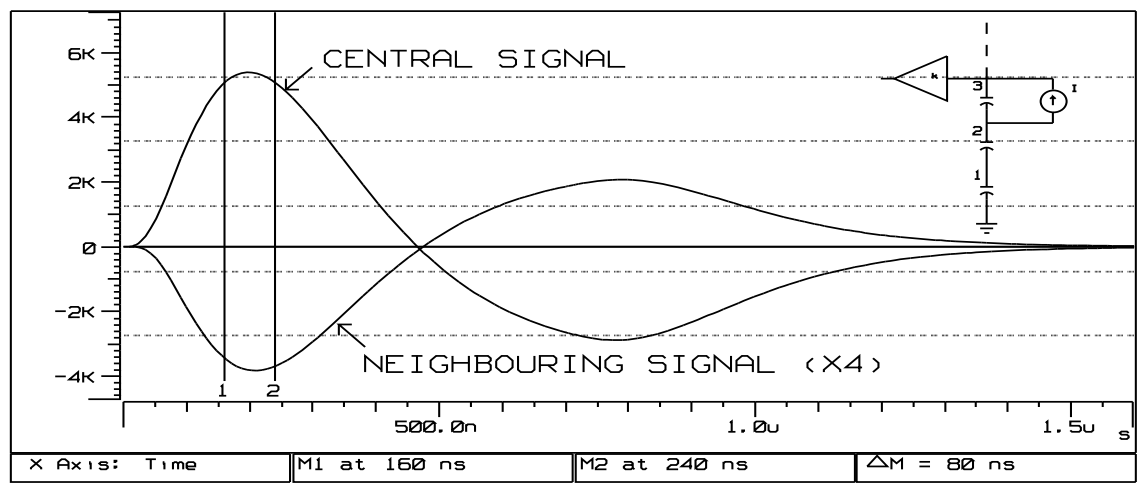

Figure 7: Simulated central tower signal and crosstalk signal in one of the adjacent cell when energy is only deposited in the gap shown. Note the change of scale between the two pulses. 


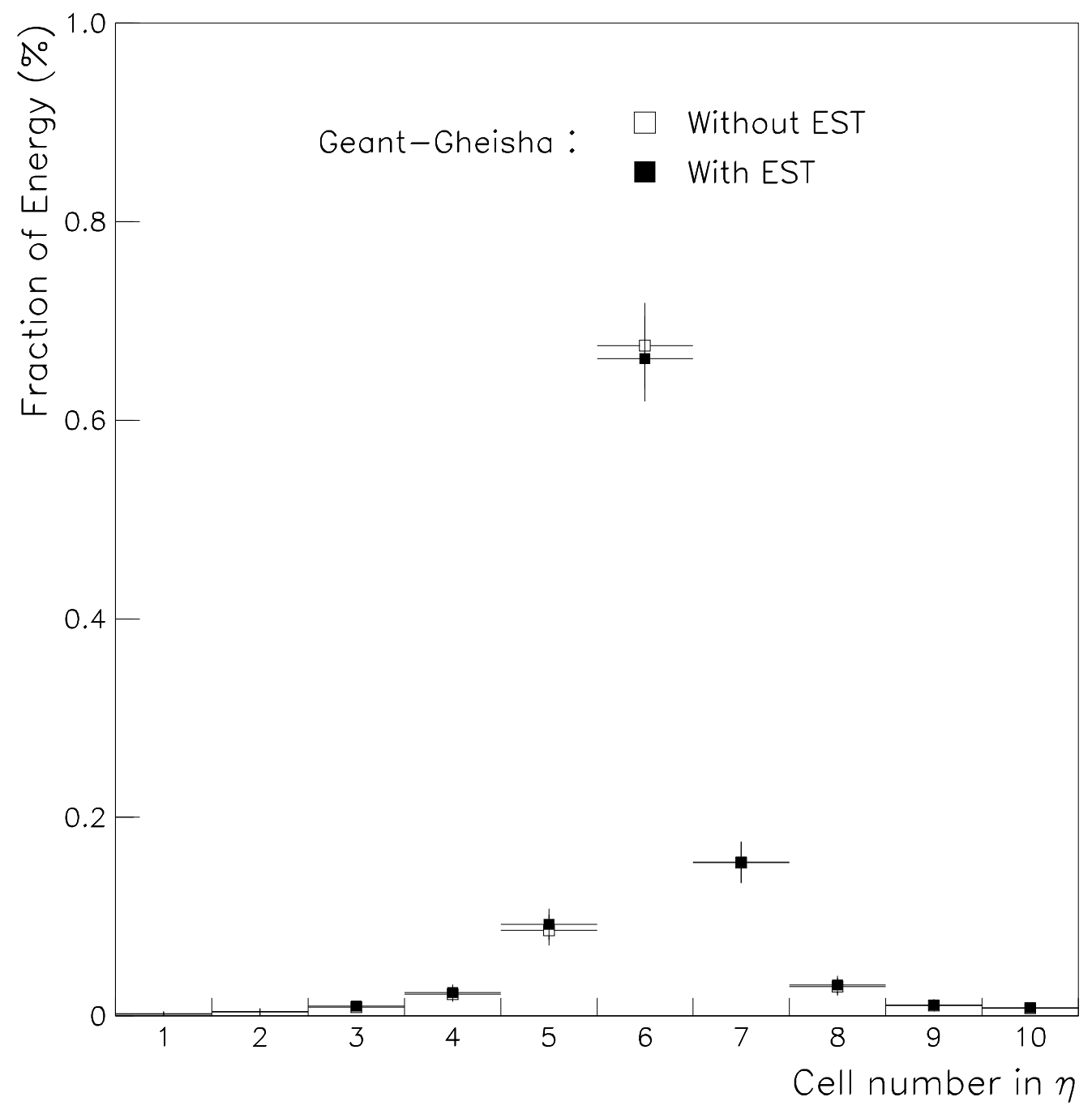

Figure 8: Transverse energy distribution in the $\eta$ direction for $100 \mathrm{GeV}$ pions in the GEANT-GHEISHA simulation with and without EST crosstalk. 


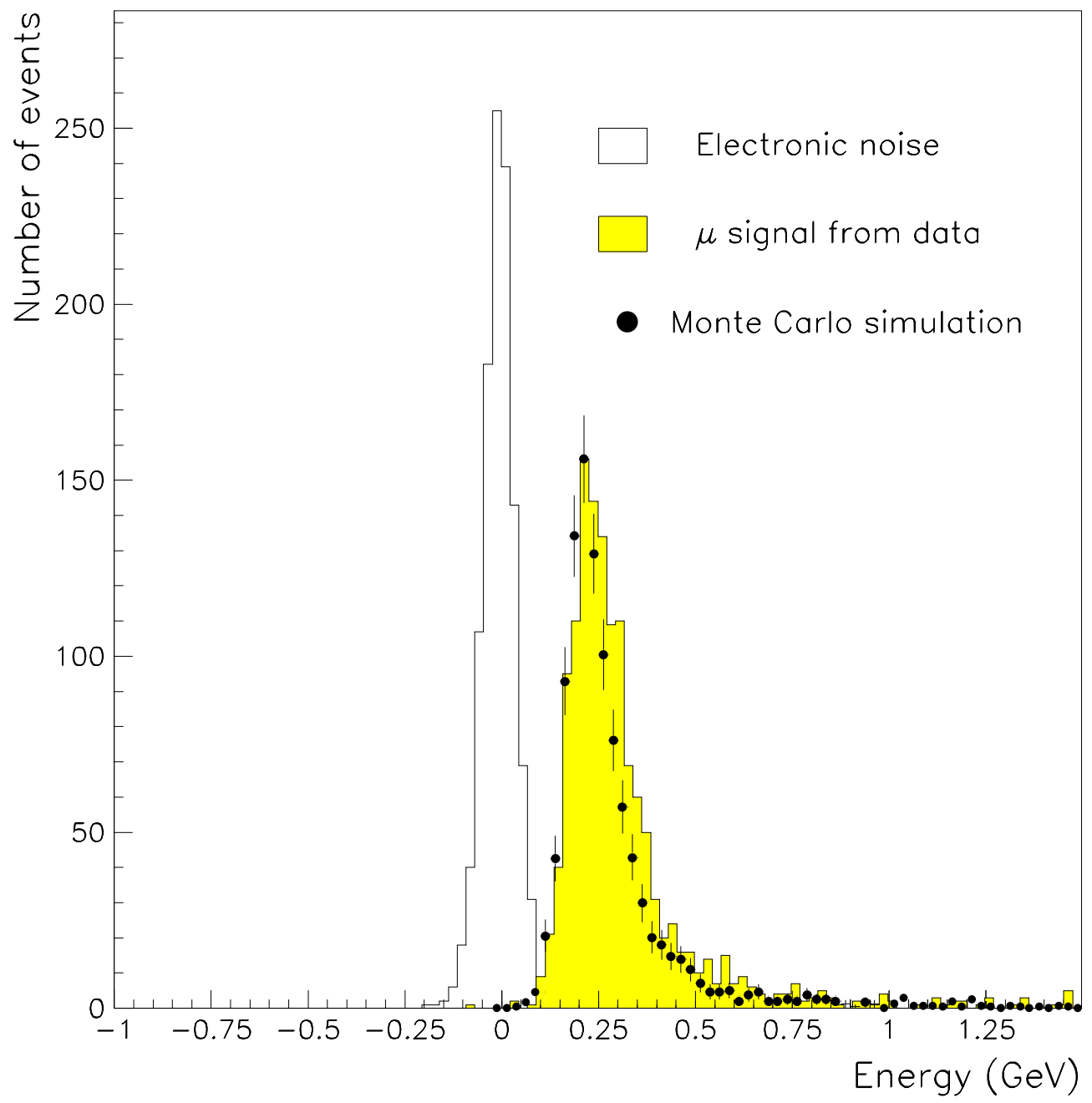

Figure 9: Energy distribution in the first sampling of the hadronic calorimeter for muons and for random trigger events. 


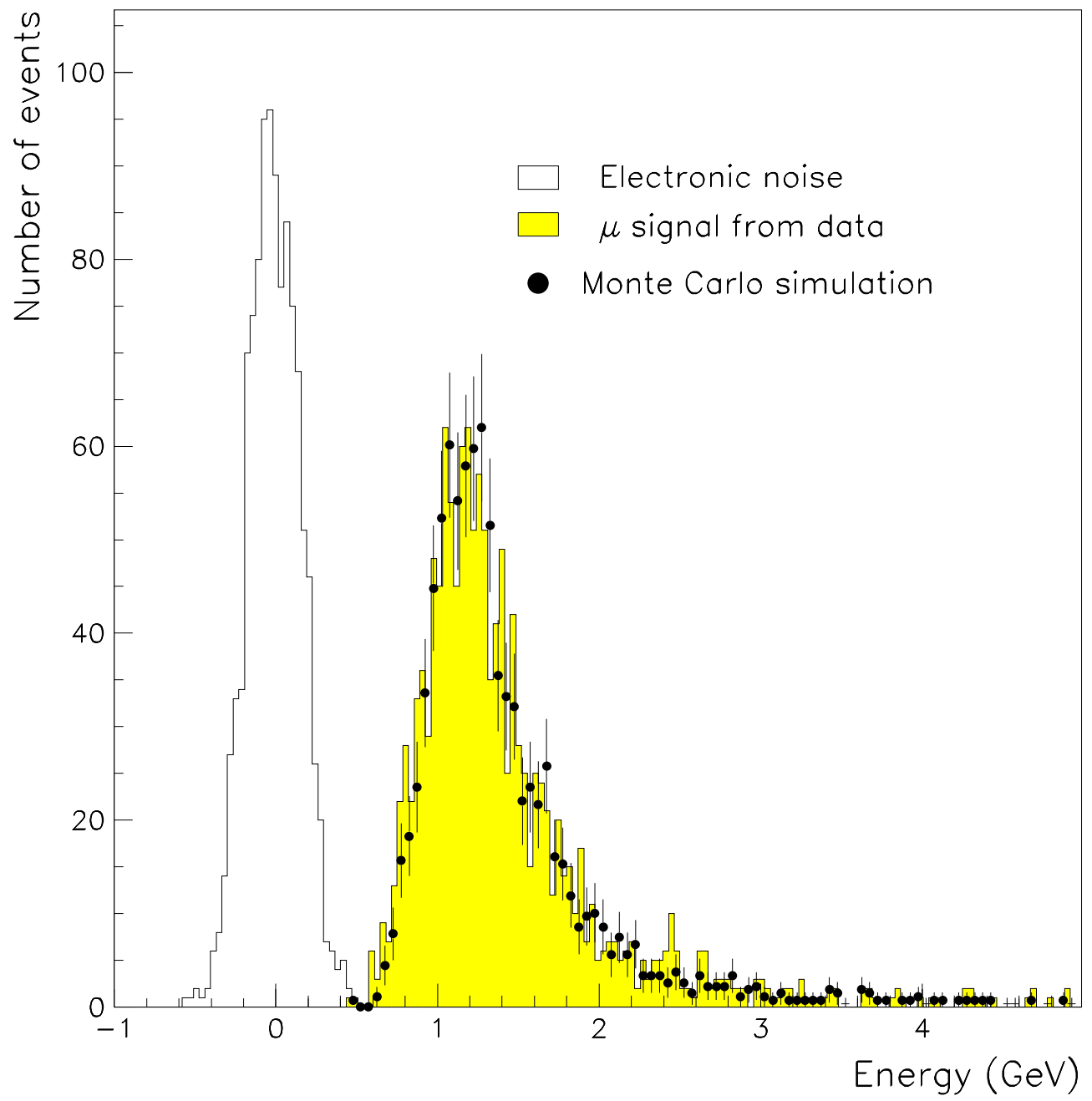

Figure 10: Energy distribution in the hadronic calorimeter over the four samplings in depth for muons and for random trigger events. 


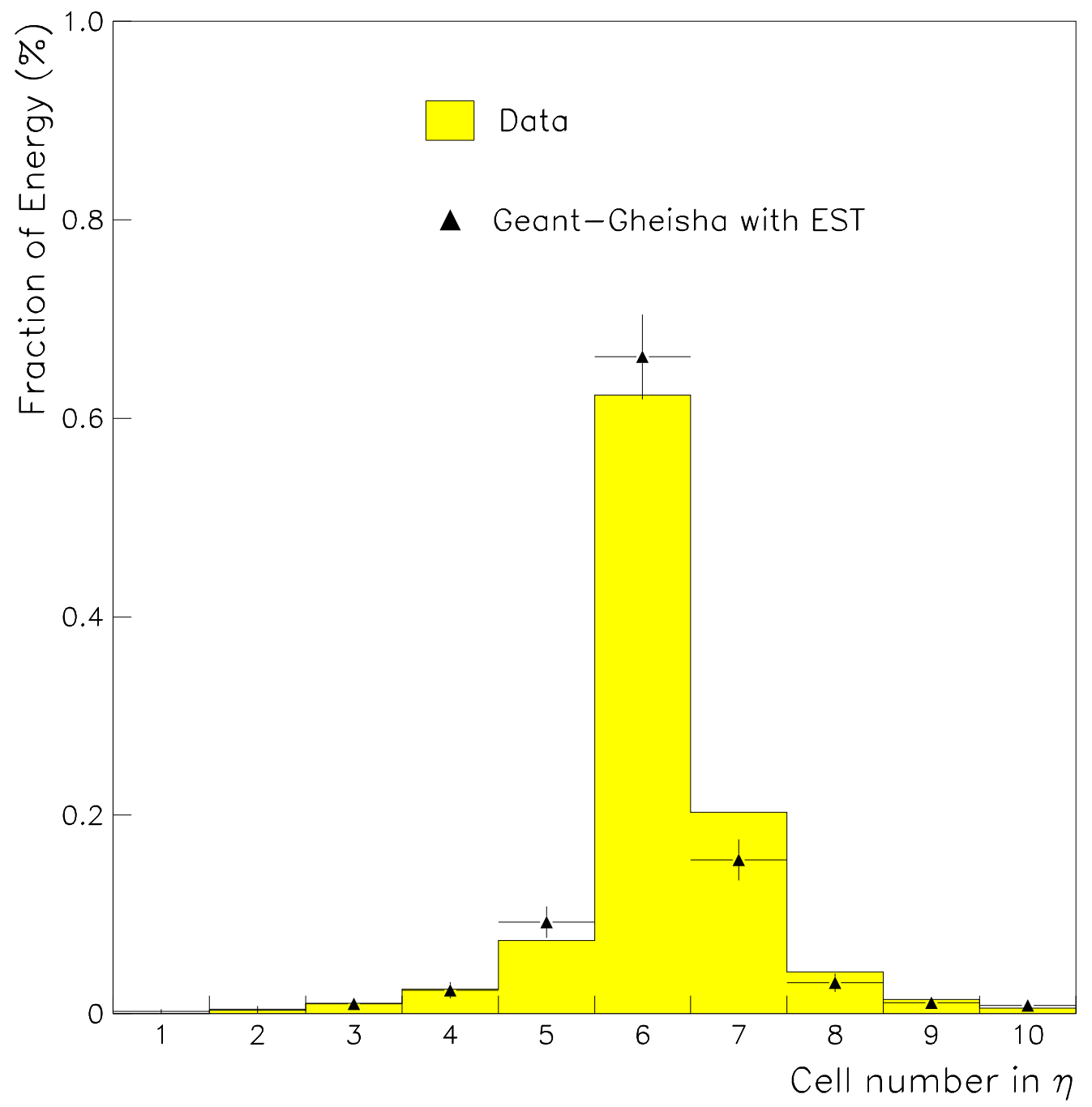

Figure 11: Transverse energy distribution in the $\eta$ direction for $100 \mathrm{GeV}$ pions. 


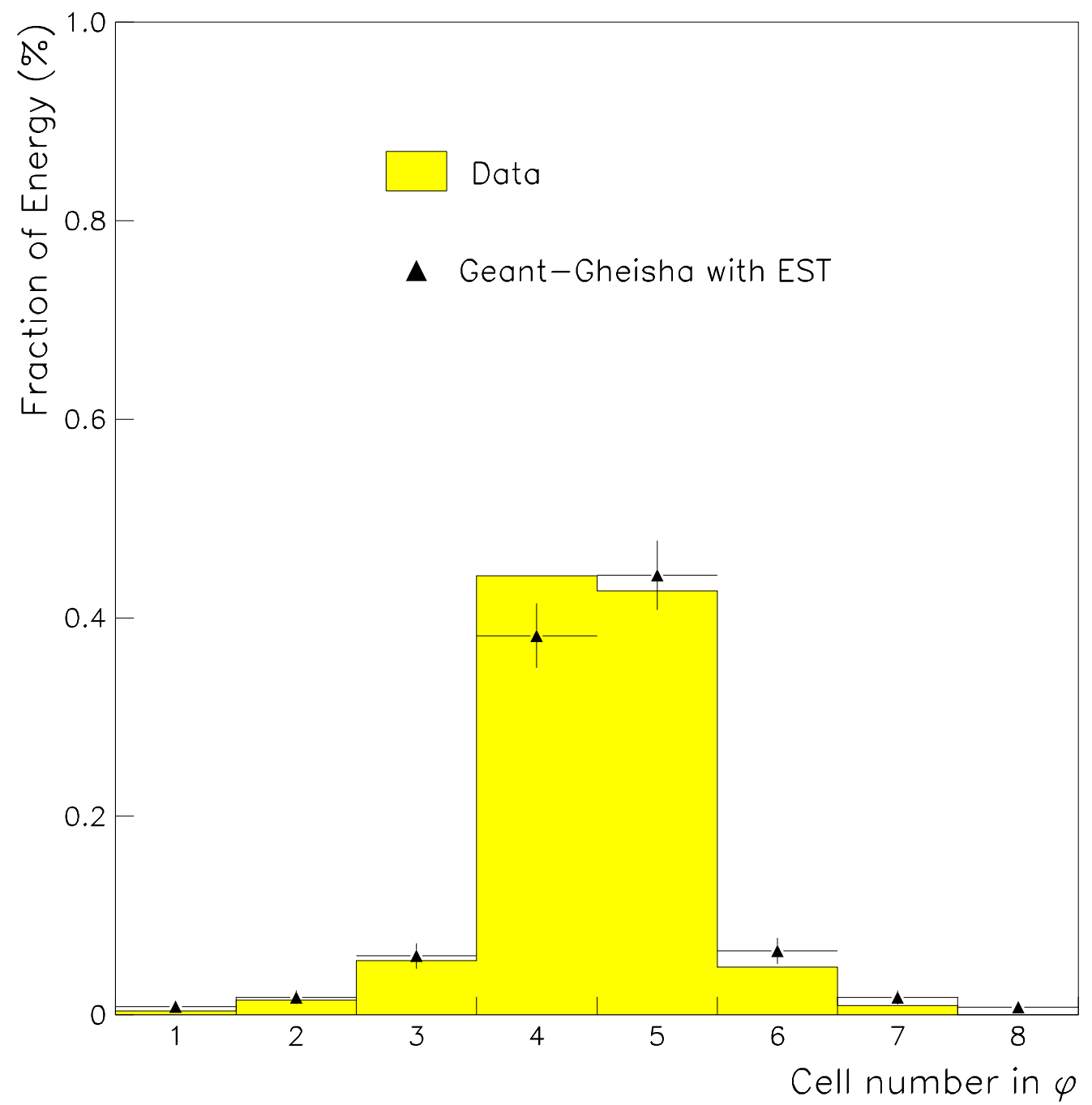

Figure 12: Transverse energy distribution in the $\phi$ direction for $100 \mathrm{GeV}$ pions. 


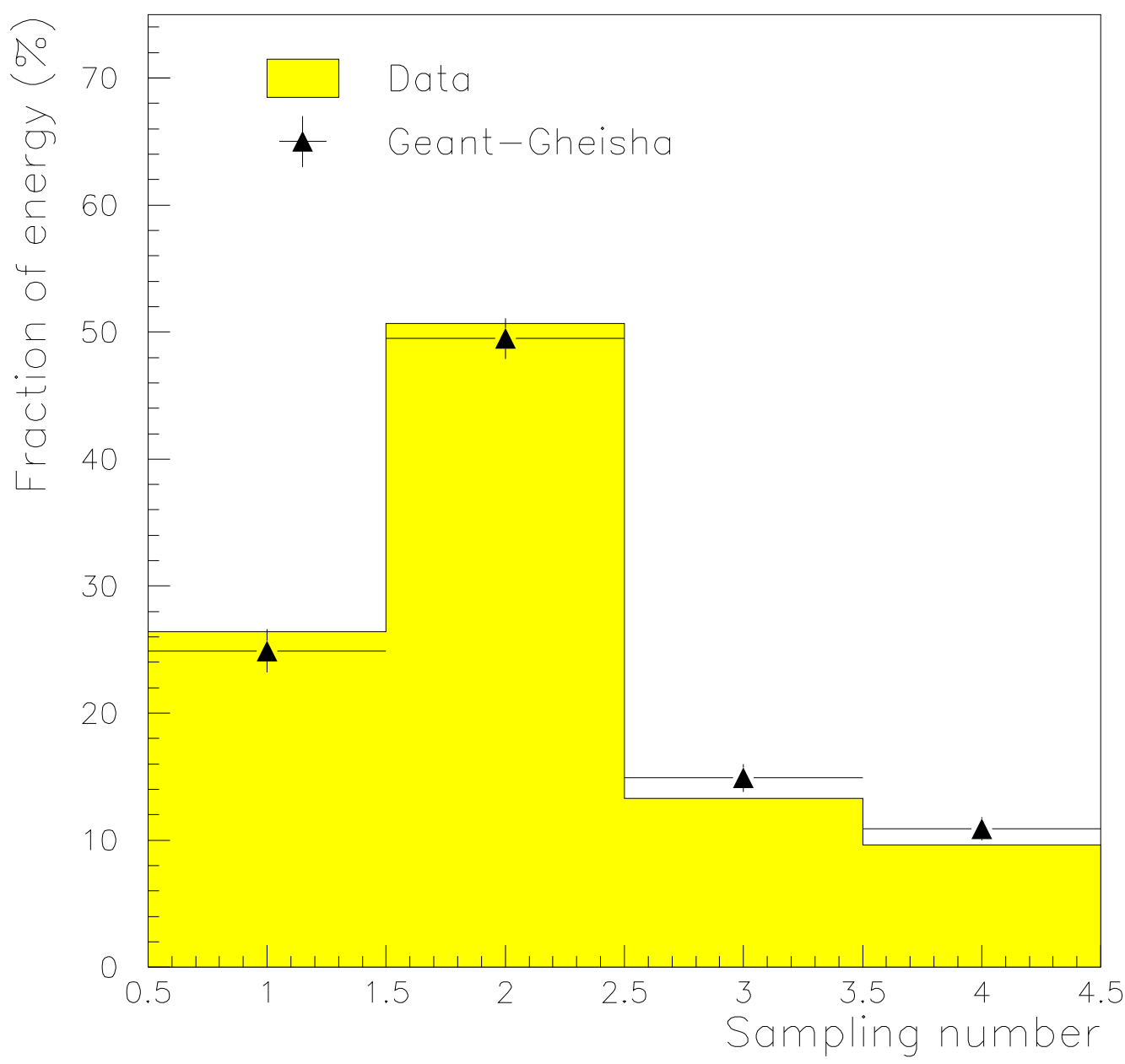

Figure 13: Longitudinal profile for $400 \mathrm{GeV}$ pions interacting in the first hadronic sampling. 


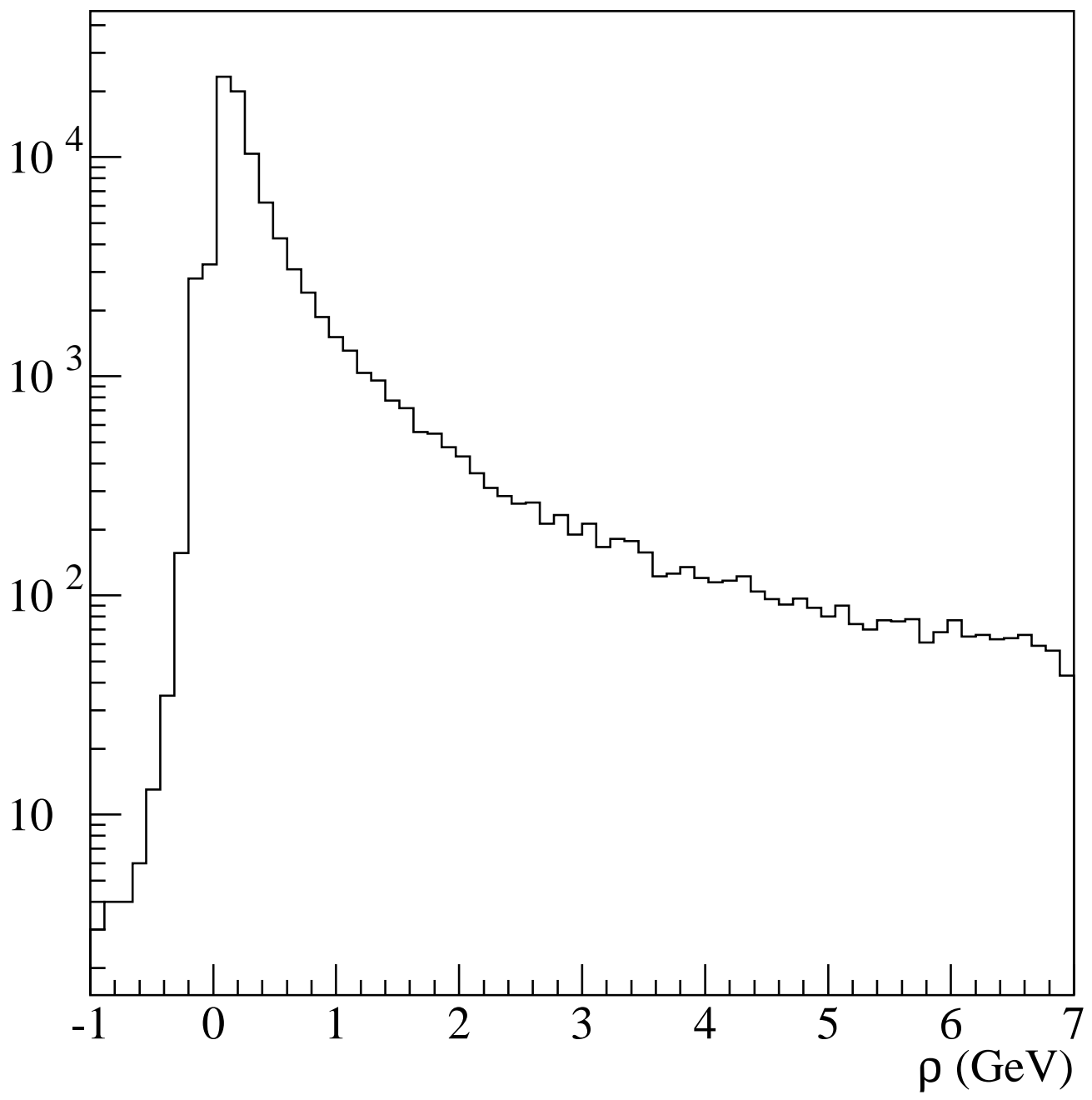

Figure 14: Distribution of the cell energy density $\rho$ for $100 \mathrm{GeV}$ pions. 


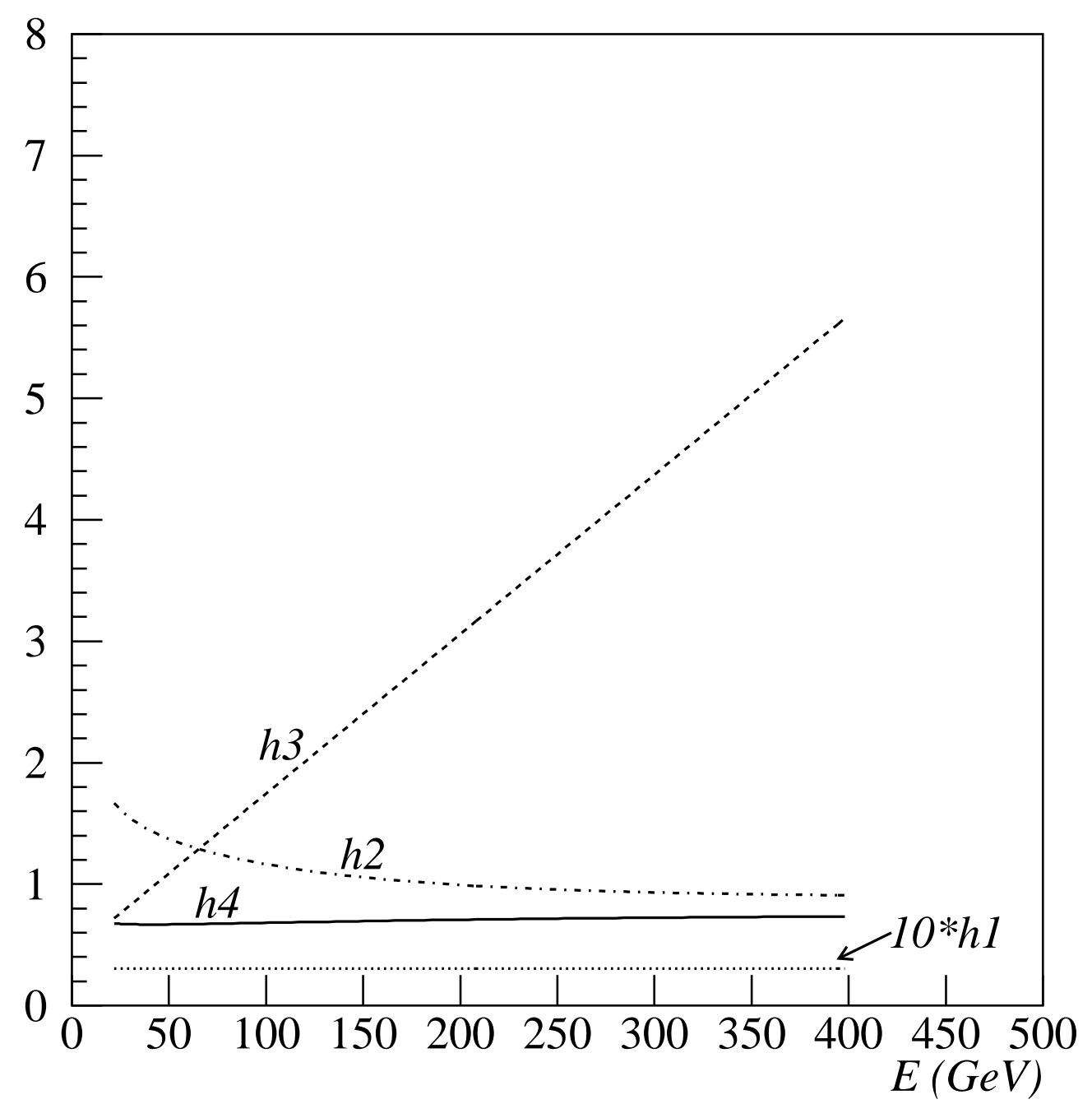

Figure 15: Parameters of the weighting function $w_{h}$ as a function of beam energy. $\mathrm{h} 1$ and $\mathrm{h} 3$ are in $\mathrm{GeV}$. 


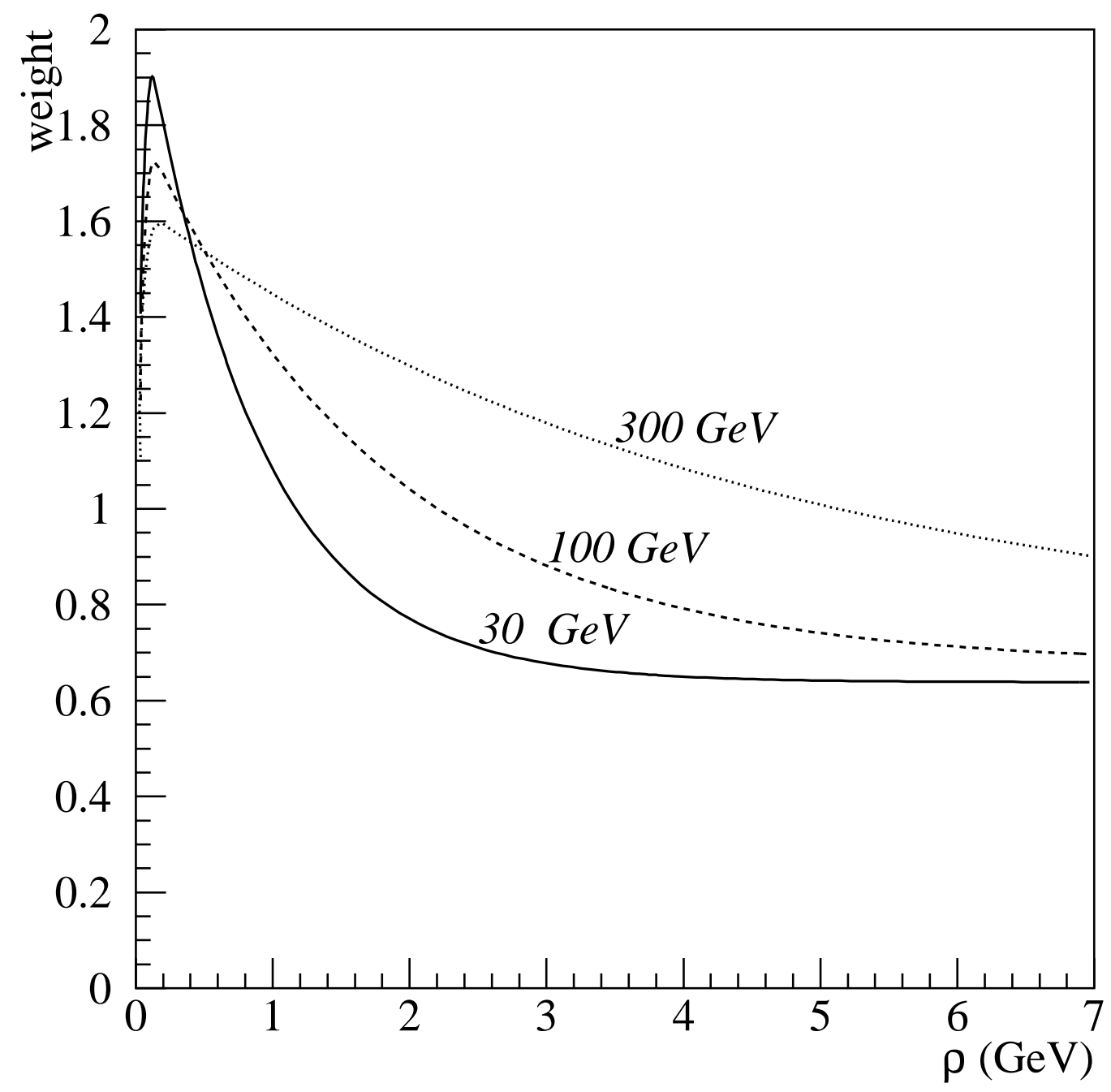

Figure 16: Weighting function $w_{H A D}(\boldsymbol{\theta}, \rho)$ in hadronic modules for several beam energies. 


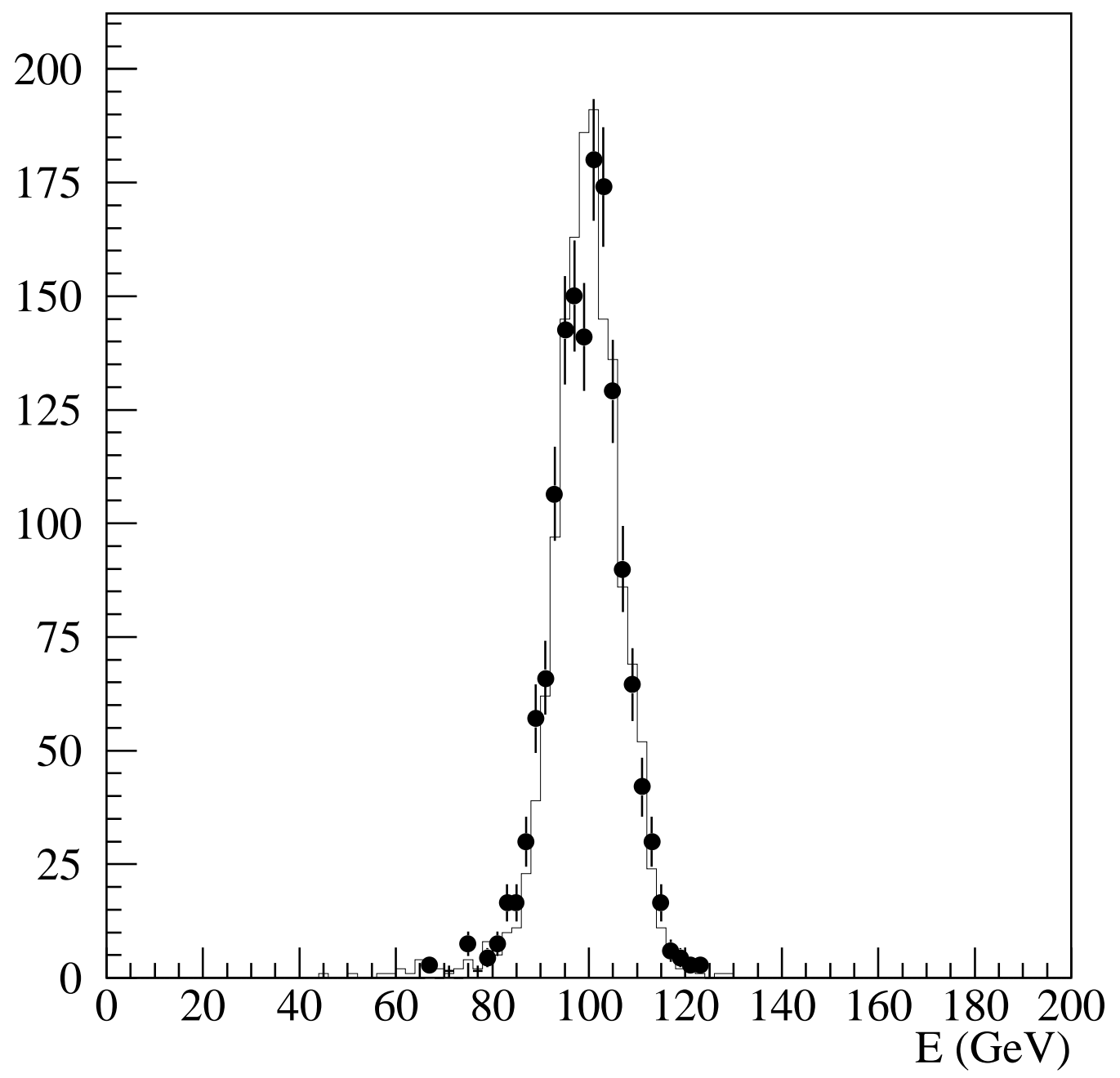

Figure 17: Distribution of the reconstructed energy for $100 \mathrm{GeV}$ pions. The histogram represents the data, dots are the GEANT-GHEISHA Monte Carlo simulation results. 


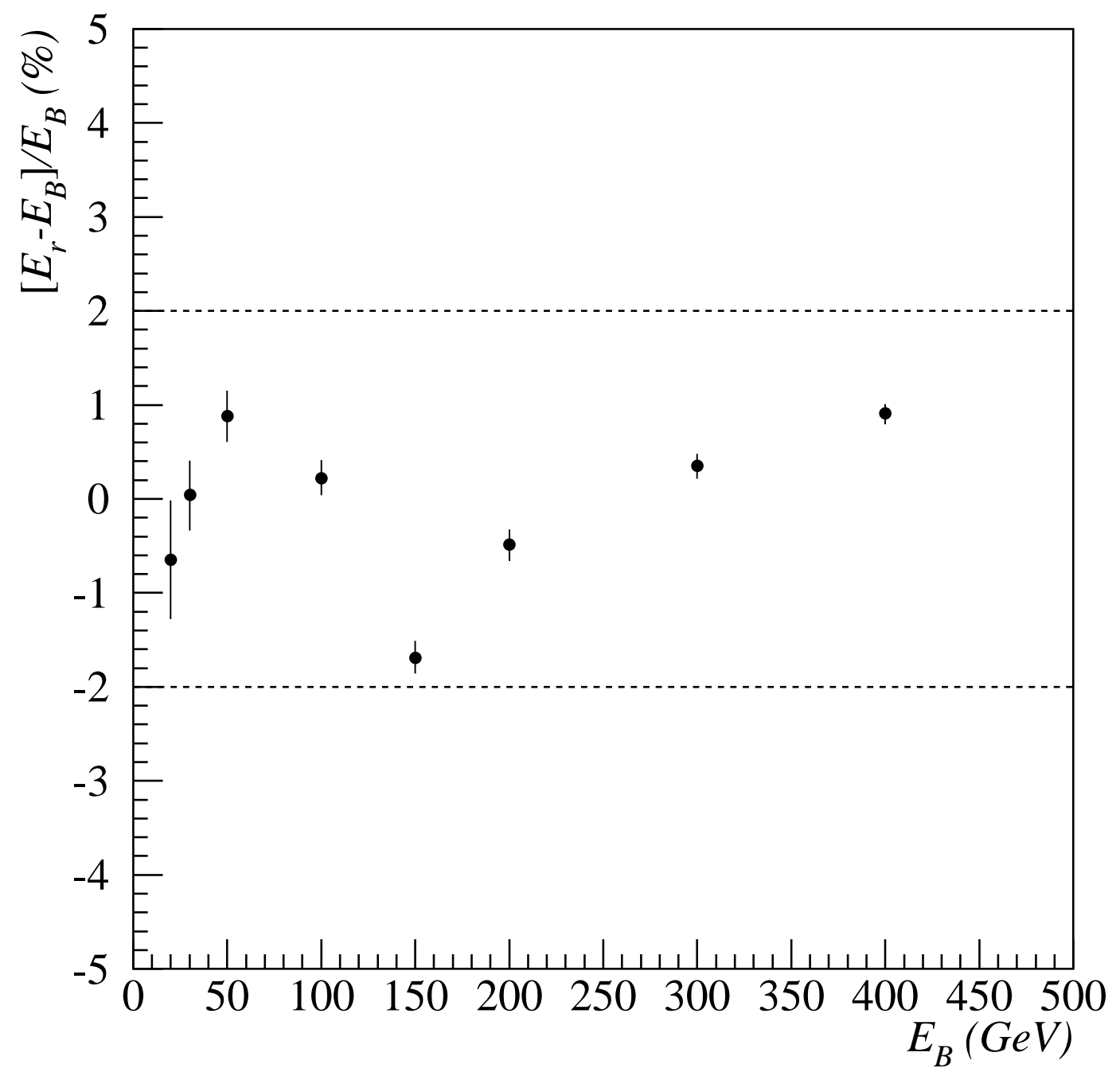

Figure 18: Relative difference between reconstructed energy and beam energy (pion data). 


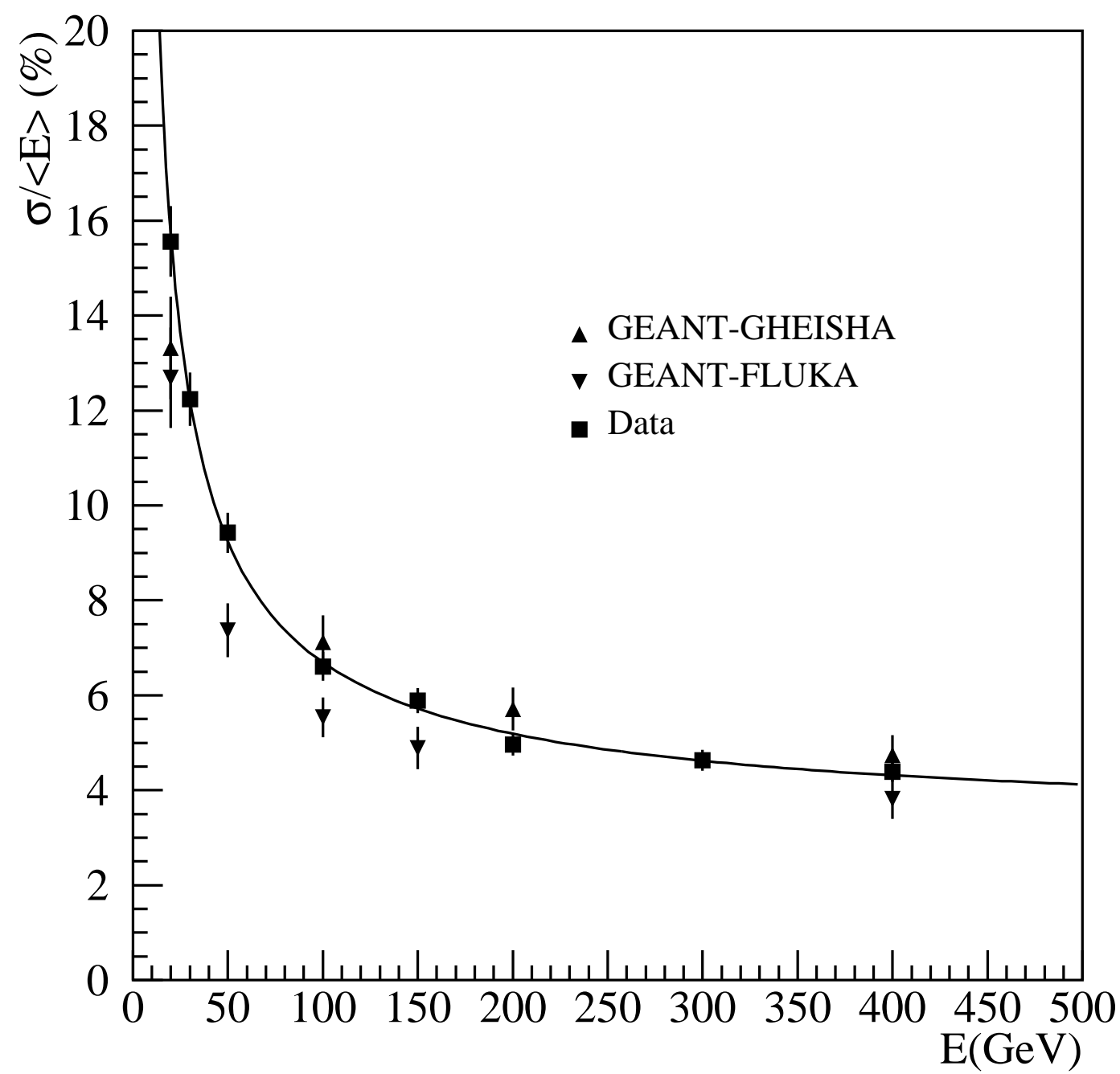

Figure 19: Energy resolution as a function of the beam energy (calibration + weighting).

Error bars are 3 standard deviation errors. An additional error obtained by varying the noise value within its error has been added to the $20 \mathrm{GeV}$ Monte Carlo points. 


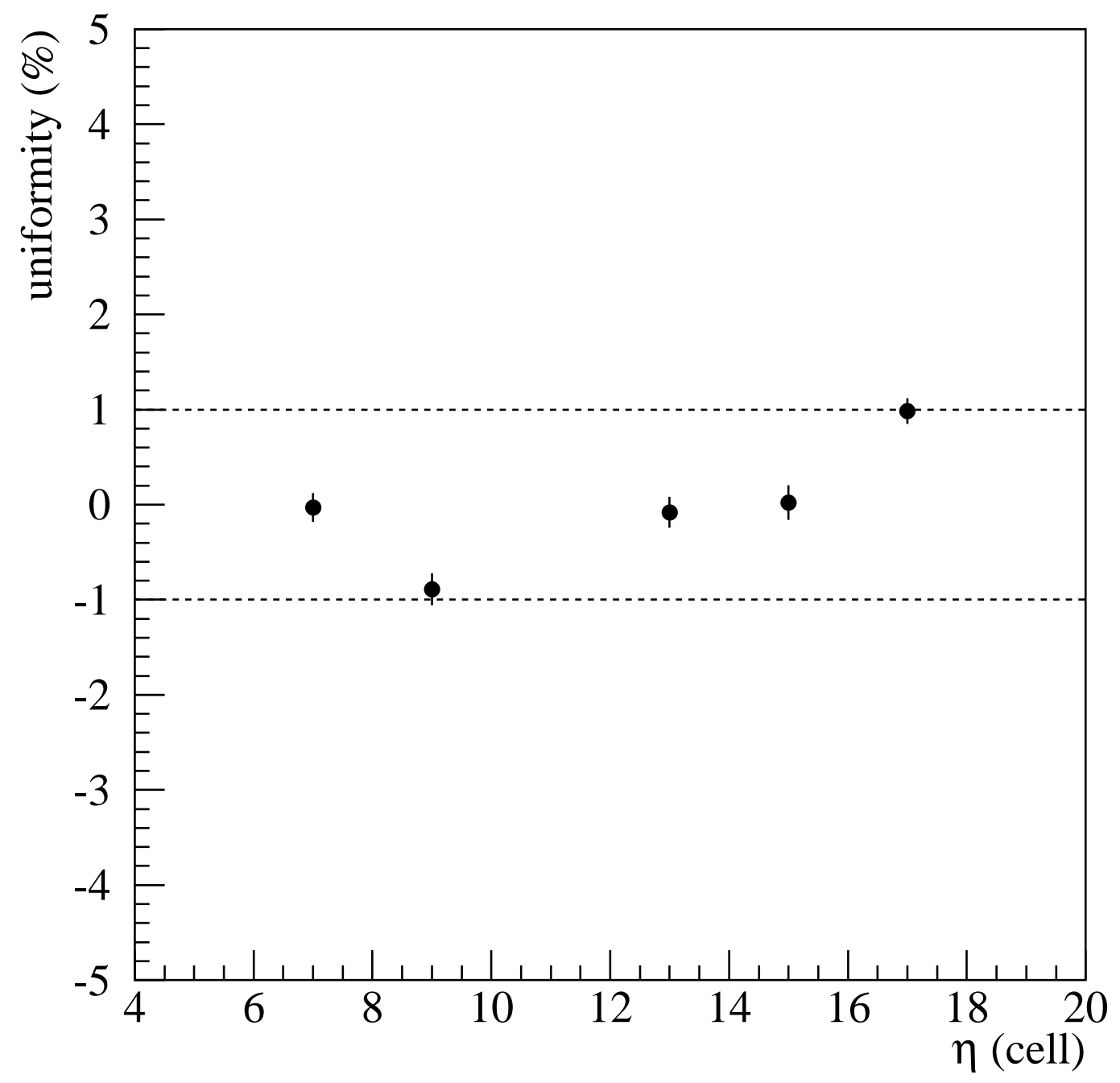

Figure 20: Uniformity of the calorimeter response as a function of impact point position. 